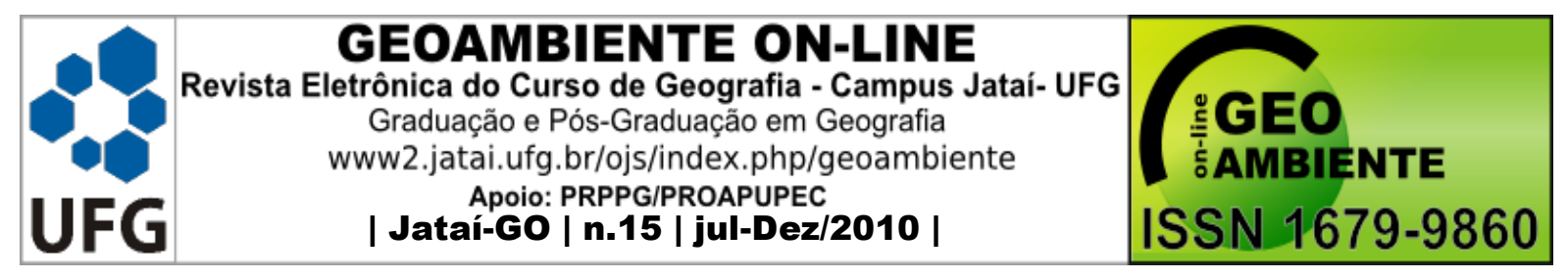

\title{
GESTÃo SUSTENTÁVEL DA PECUÁRIA NA BACIA DO RIO SAN PEDRO, EM CAMAGÜEY, CUBA
}

\author{
Zoe Griselda Acosta Gutiérrez ${ }^{1}$, Guillermo Guevara Viera²
}

(1.- Centro de Investigaciones de Medio Ambiente de Camagüey, Cuba. Cisneros No. 105 (altos) e/ Ángel y Pobre. C.P. 70100 Camagüey, Cuba. Telefax: (53) (32) 298268. Investigadora Auxiliar, Jefe del Dpto. de Biodiversidad. Dirección de correo electrónico: zoe@cimac.cu; 2.- Universidad de Camagüey, Facultad de Ciencias Agropecuarias, Centro de Estudios para el Desarrollo de la Producción Animal. Carretera Circunvalación Norte Profesor Auxiliar. Dirección de correo electrónico: guillermo.guevara@ reduc.edu.cu)

\section{Resumen}

\section{ORDENAMIENTO SOSTENIBLE DE LA GANADERÍA BOVINA EN LA CUENCA HIDROGRÁFICA DEL RÍO SAN PEDRO EN CAMAGÜEY, CUBA.}

Para lograr un ordenamiento sostenible de la ganadería bovina en la cuenca hidrográfica del río San Pedro en Camagüey, Cuba, se aplicó la secuencia: diagnóstico ambientalcaracterización-agrupamiento multifactorial-propuesta de alternativas y evaluación de sus impactos. La caracterización de la actividad (40 entidades) que, incluyó el análisis de 53 variables relacionadas con la disponibilidad de recursos y el comportamiento de indicadores productivos y reproductivos, puso de manifiesto una elevada heterogeneidad entre entidades y un indicador general de impacto ambiental muy alto (- 1 094), ocasionado por la ganadería bovina a la cuenca bajo estudio. En el análisis multifactorial empleado para los agrupamientos, se encontró que las entidades de la parte alta, formaron tres grupos bien definidos, mientras que en la parte baja, se crearon cuatro grupos de entidades. La descripción de las agrupaciones se utilizó para la propuesta de las alternativas que se deben poner en práctica, con vistas a mitigar los impactos identificados, lo que se desarrolló con técnicas participativas. Se concluye que el conjunto de alternativas evaluado para cada agrupación, reduciría el impacto ambiental ocasionado por la ganadería a la cuenca y que el ordenamiento que se aplica, puede encaminar la actividad económica hacia la sostenibilidad.

Palabras claves: ordenamiento ganadero, impacto ambiental, cuenca hidrográfica.

\footnotetext{
Artigo recebido para publicação em 07 de julho de 2010

Artigo aprovado para publicação em 18 de Dezembro de 2010
} 


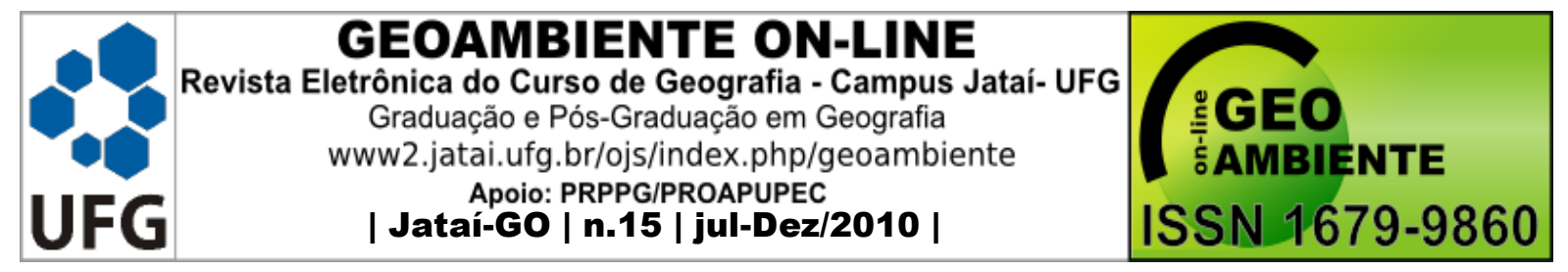

\section{Abstract \\ SUSTAINABLE MANAGEMENT OF LIVESTOCK IN THE BASIN OF SAN PEDRO RIVER IN CAMAGUEY, CUBA.}

It was applied the sequence: environmental assessment, characterization, clustering multifactorial, proposed alternatives and evaluation of its impact, in order to achieve sustainable management of livestock in the basin of San Pedro River in Camaguey, Cuba. The characterization of the activity (40 entities), that included analysis of 53 variables related to resource availability and the performance of productive and reproductive indicators, revealed a high heterogeneity between banks and a general indicator of environmental impact is too high ( - 1094) caused by cattle to the basin under study. The multivariate analysis used for the cluster, we found that entities in the upper part, formed three distinct groups, while in the lower part, four groups were created entities. The description of the groups was used for the proposed alternatives to be implemented in order to mitigate the identified impacts, which was developed participatory techniques. We conclude that the set of alternatives evaluated for each group, reduce the environmental impact caused by livestock in the basin and that the order applies, you can steer the economy towards sustainability.

Keywords: livestock management, environmental impact basin.

\section{Resumo}

Para alcançar uma gestão sustentável da pecuária na bacia hidrográfica do Rio São Pedro, em Camaguey, Cuba, se aplicou em sequencia: um diagnóstico ambiental, caracterização, agrupamento multifatorial, propostas alternativas e avaliação dos seus impactos. A caracterização da atividade (40 entidades), que incluiu a análise de 53 variáveis relacionadas avaliou a disponibilidade de recursos e o desempenho dos indicadores produtivos e reprodutivos. Os resultados revelaram uma grande heterogeneidade entre os dados e um indicador geral do impacto ambiental muito elevado (- 1094) causado pela pecuária na bacia em estudo. Na análise multivariada utilizada para os grupos, descobriu-se que as entidades na parte superior, eram formadas por três grupos distintos, enquanto na parte inferior, foram agrupados em quatro grupos de entidades. Na descrição dos grupos foi utilizado propostas alternativas a serem implementadas a fim de mitigar os impactos identificados, que foi desenvolvido técnicas participativas. Concluí-se que o conjunto de alternativas avaliadas para 


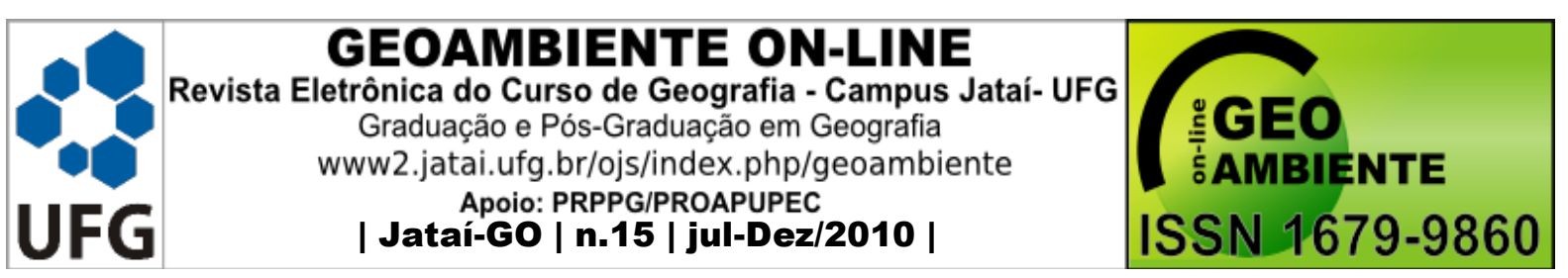

cada grupo, reduziria o impacto ambiental causado pela pecuária na bacia e que o manejo que se aplica, pode guiar a economia da área em estudo para a sustentabilidade.

Palavras-chave: manejo da pecuária; da bacia de impacto ambiental.

\section{Introducción}

En Cuba, al igual que en muchos países de América Latina, el reto que afronta el desarrollo de la producción ganadera en las actuales condiciones de deterioro en que se encuentran los principales recursos naturales disponibles, presupone no sólo la aplicación de los resultados obtenidos como parte del desarrollo científico-tecnológico del sector, sino también, la consideración de las particularidades de cada ecosistema para la aplicación de las alternativas de manejo correspondientes, aspecto bien identificado y discutido en las Estrategias Ambientales del país (CUBA, 1997; 2006).

En línea con lo anterior, en las últimas décadas se ha trabajado intensamente en la reconversión del ambiente pecuario cubano sin embargo, se aprecia la necesidad de perfeccionar y extender una concepción más integral.

En este sentido, y de acuerdo con el criterio de diferentes autores de la región y el país (DOUROJEANNI, 1997; FRÓMETA et al., 2004; VILLASUSO, 2004; IBRAHIM et al., 2006), se reconoce que la cuenca hidrográfica es un espacio adecuado para la planificación de las acciones que se deben acometer, por cuanto a ese nivel se puede lograr una visión más amplia de las causas de las intervenciones del hombre sobre los recursos naturales así como, los efectos de su actividad sobre dichos recursos y su repercusión sobre la producción y el bienestar de las comunidades.

También se acepta, que la planificación de dichas acciones, debe tener su base en la selección adecuada de los sistemas de manejo, las técnicas y las tecnologías que se han de aplicar, atendiendo a las características particulares de las entidades, las cuales además se deberán agrupar o clasificar para diferenciar las prácticas a seguir y garantizar un nivel de prioridad en la toma de decisiones (GUEVARA, 2005; HARVEY et al., 2005).

Lo anterior indica, que en el ordenamiento del desarrollo ganadero sustentable, se deben considerar ambos criterios sin embargo, los trabajos desarrollados al respecto, generalmente no lo asumen de igual forma.

En la microcuenca Mesa de Escalante en Guanajuato, Méjico, REYES (1999) hizo una propuesta de manejo pecuario para el desarrollo sostenible de la actividad ganadera, en la cual 


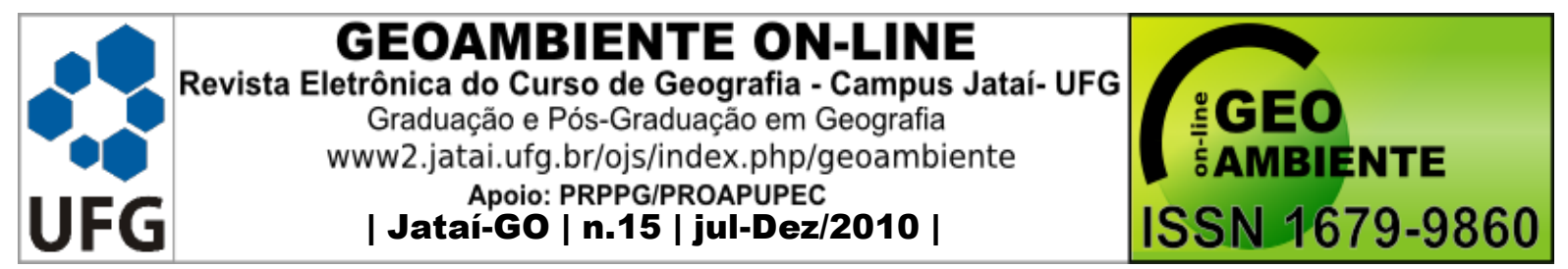

tuvo en cuenta las características ambientales de la región pero no asumió la clasificación de las fincas para la precisión de las acciones.

En la Base Aérea de Marandúa, región natural de la Orinoquia colombiana, BUSTAMANTE (2002) desarrolló un sistema de clasificación como estrategia para la planificación de sistemas agroforestales y sólo consideró variables ambientales, sin analizar la actividad socio - productiva.

En la Amazonía colombiana, RAMÍREZ (2002) planteó una propuesta de alternativas productivas en fincas, para la cual clasificó previamente las entidades ubicadas en el contexto de la cuenca hidrográfica sin incluir variables relativas al comportamiento productivo y reproductivo de los animales en explotación.

En Cuba, GUEVARA (2005) clasificó las entidades ganaderas ubicadas en una región de alta densidad en la producción de leche, la cual denominó cuenca lechera, pero omitió algunos aspectos básicos, relativos a las cuencas hidrográficas en las que dichas entidades desarrollan sus actividades.

Por otra parte, los diagnósticos de cuencas los cuales constituyen instrumentos de gran valor para la gestión integrada, no logran la profundidad que merece el ordenamiento de una ganadería sostenible, incluso a la luz de la nueva metodología propuesta por la DIRECCIÓN NACIONAL DE CUENCAS HIDROGRÁFICAS (2006).

Lo anterior revela la necesidad de establecer una secuencia de trabajo que propicie el ordenamiento de esta actividad económica, en un contexto de cuenca hidrográfica, con vistas a lograr la sostenibilidad. El objetivo del presente trabajo consistió en aplicar la secuencia diagnostico ambiental-caracterización-clasificación-evaluación de propuestas de mitigación en el ordenamiento sostenible de la ganadería bovina, en la cuenca hidrográfica del río San Pedro en Camagüey, Cuba.

\section{2.- Materiales y métodos generales}

\subsection{Localización}

La cuenca hidrográfica del río San Pedro se localiza en la vertiente sur de la provincia de Camagüey, entre las coordenadas 272000 y 316000 Norte y las 326000 y 404000 Este, Proyección Cónica Lambert Cuba Sur. Limita al norte, con las cuencas hidrográficas de los ríos Máximo y Saramaguacán, al oeste con la de los ríos Caonao y La Yeguas y al este con la del río Najasa (PRIMELLES et al., 2003). 


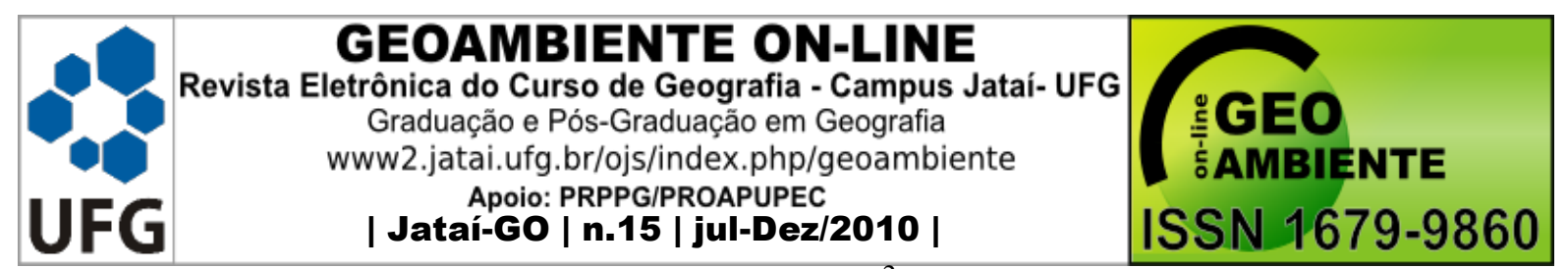

La extensión de la cuenca es de $1053 \mathrm{~km}^{2}$, tiene una población de unos 319000 habitantes (no incluye la población dispersa), y abarca territorios de los municipios Camagüey, Jimaguayú y Vertientes. En ella se localiza la ciudad de Camagüey, segunda del país por su extensión y tercera por la cantidad de habitantes (unos 308 000), y la presa Jimaguayú una de las mayores y más productivas del país, entre otros aspectos.

Los principales tenentes de la tierra en esta región son: la Empresa Pecuaria de Vertientes con 16072 ha, la Empresa Pecuaria Triángulo 5 con 15411 ha el CAI Arrocero Ruta Invasora con 11332 ha, la Empresa Genética Rescate de Sangüily con 10947 ha, la Empresa Pecuaria. Triángulo 1 con 9292 ha, la Empresa Pecuaria Triángulo 3 con 8161 ha, y el Sector Campesino con 5607 ha, lo que representa el 62.9\% del área total (ACOSTA et al., 2006).

\section{2.- Procedimientos y métodos}

El trabajo se hizo en tres etapas: caracterización de la actividad ganadera, clasificación de las entidades productivas y evaluación del impacto ambiental que ocasionaría el establecimiento de alternativas de manejo zootécnico, para mitigar los impactos identificados en la primera etapa.

\subsubsection{Caracterización}

Para caracterizar la ganadería bovina e identificar los impactos que ocasiona, se hicieron observaciones directas en recorridos de campo y se tomó información estadística actualizada (28 variables), acerca del estado de los principales componentes de la naturaleza, la producción ganadera y el personal vinculado a dicha actividad, en las 40 entidades (estructura básica de producción) cuyo objeto social se vincula a la producción bovina en la región.

Para el cálculo de los estadígrafos se utilizó el paquete estadístico SYSTAT 10.2 (2002).

La valoración del impacto ambiental se hizo a juicio de expertos, de acuerdo con el Método de GÓMEZ OREA (1999), atendiendo a la siguiente fórmula:

$$
\mathrm{I}=(3 \mathrm{I}+2 \mathrm{E}+\mathrm{M}+\mathrm{P}+\mathrm{R}) \quad \text { donde: }
$$

II= Importancia del impacto

I= Intensidad: representa el grado de modificación del componente. 


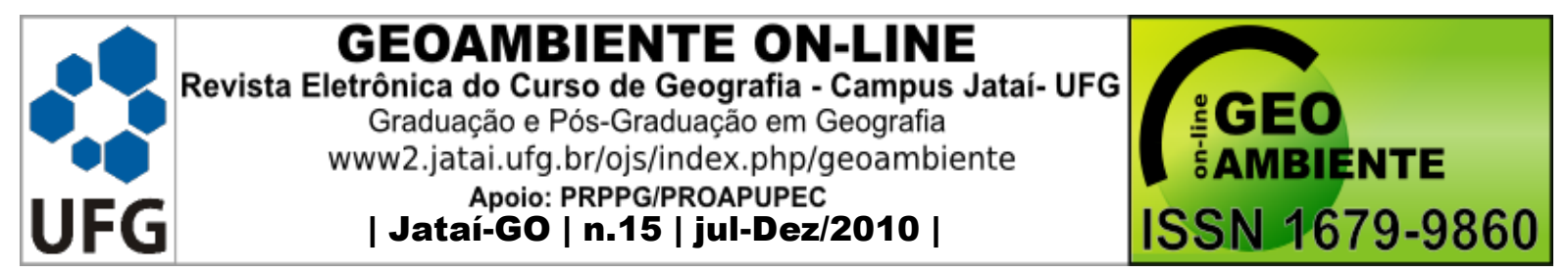

E= Extensión: área de influencia del impacto.

M= Momento: plazo de manifestación del impacto.

$\mathrm{P}=$ Persistencia: tiempo de permanencia del efecto de impacto.

$\mathrm{R}=$ Reversibilidad: posibilidad de recuperación del factor afectado.

El grado de afectación de la cuenca se determinó según la siguiente escala:

MUY ALTO: [> 210]; ALTO: [160-210]; MEDIO: [120-160]; BAJO: [60-120]; MUY BAJO: $[<60]$

Las componentes que se consideraron para el análisis fueron:

\begin{tabular}{llr}
\multicolumn{2}{c}{ Subsistema Naturaleza } & $\begin{array}{c}\text { Subsistema Socioeconomía } \\
\text { I. }\end{array}$ \\
II. & Geomorfología & Atmósfera \\
III. & Hidrología & \\
IV. & Suelo & \\
V. & Vegetación \\
VI. & Fauna & \\
VII. & Paisaje
\end{tabular}

\subsubsection{Clasificación}

La información primaria relacionada con el comportamiento de las principales variables climáticas en la región (PRIMELLES et al., 2003), reporta que existen diferencias entre la parte alta (PA) y la parte baja de la cuenca (PB). Por la estrecha relación que tiene el clima con la productividad de los sistemas ganaderos tropicales (JEREZ, 1983) y por su participación en el sistema integrado clima-calidad de los pastos-comportamiento animal (SENRA, 2001); en este estudio, se asumió la condición de agrupar las entidades productivas dentro de la localidad en que se ubican, según su posición en relación con la coordenada 290 000 Proyección Cónica Conforme de Lambert Cuba Sur.

La clasificación se hizo a través del análisis estadístico k-means clustering (SYSTAT 10.2., 2002), que particiona en grupos disjuntos. Las variables fueron previamente estandarizadas con medía 0 y desviación estándar 1 . Se hizo un primer cluster en cada localidad, para discriminar aquellas variables que no aportaban a la formación de los grupos y luego se procedió al agrupamiento, sólo con las variables que aportaban al análisis, las que fueron: AREAT= área total (ha), AREAM= área con Marabú (ha), PASTOSA= área de pastos 


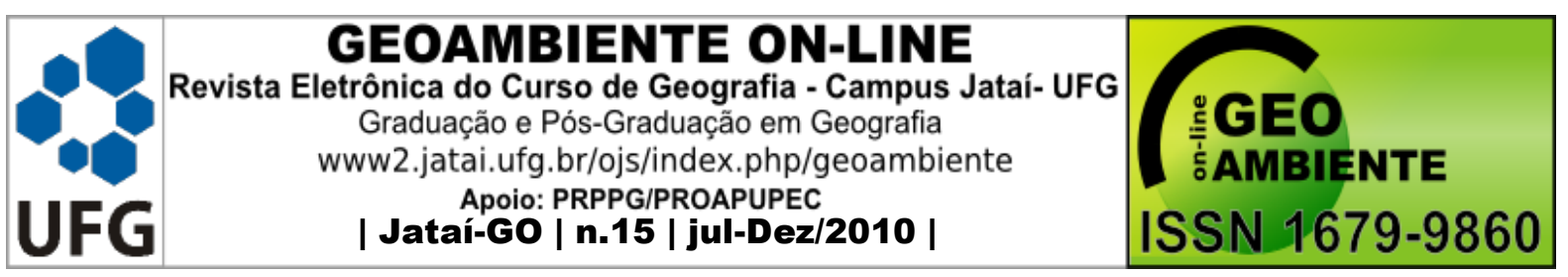

cultivados (ha), PASTON= área de pastos naturales (ha), NOARB= especies de árboles en potreros $(\mathrm{No}), \mathrm{FORR}=$ área de forrajes $(\mathrm{ha}), \mathrm{TT}=$ total de trabajadores $(\mathrm{No}), \mathrm{TEC}=$ técnicos $(\mathrm{No}), \mathrm{DIRIG}=$ dirigentes $(\mathrm{No}), \mathrm{OBRE}=$ obreros $(\mathrm{No}), \mathrm{SERV}=$ trabajadores de servicios $(\mathrm{No})$, $\mathrm{POZO}=\operatorname{pozos}(\mathrm{No}), \mathrm{MOLINO}=\operatorname{molinos}(\mathrm{No}), \mathrm{TRANQUE}=$ tranques $(\mathrm{No}), \mathrm{MICRO}=$ micro presas $($ No), NSAT $=$ nivel de satisfacción de agua $(\%), \mathrm{VACA}=$ total de vacas $(\mathrm{No})$, SUELOE = niveles de erosión del suelo (1-4) PLECHE= producción de leche (L), NAC $=$ nacimientos $($ No), MUERTE $=$ muertes $($ No), GASTOSA $=$ gastos de salario (\$),GASTOT=gastos totales $(\$), \mathrm{GASTOPP}=$ costo por peso $(\$)$, PLXHA= producción de leche.ha ${ }^{-1}(\mathrm{~L})$, NACXHA $=$ Nacimientos.ha ${ }^{-1}$ (No), GASALXHA= gastos de salario.ha ${ }^{-1}$ (\$),GASTOTXHA = gastos totales.ha ${ }^{-1}(\$)$, PLXVACA = Producción de leche.vaca ${ }^{-1}(\mathrm{~L})$, PLXTT $=$ Producción de leche. total de trabajadores ${ }^{-1}$ (L), PLXOBRE= Producción de leche . obrero $^{-1}$ (L) y NACXVAC $=$ Nacimientos.vaca ${ }^{-1}$ (No). Se determinaron los estadígrafos fundamentales por grupos y se describieron los mismos.

\subsubsection{Evaluación de impacto ambiental de las alternativas de mitigación que se proponen}

Las propuestas de acciones de manejo zootécnico a realizar en cada agrupación de entidades (siete grupos: tres ubicados en la parte alta de la cuenca y cuatro en la parte baja), se lograron con un ejercicio participativo. El procedimiento que se utilizó en éste, siguió las pautas propuestas por BEAULIE et al. (2000) y se condicionó a una visión futura de cinco años.

En la evaluación de los impactos que ocasionaría la aplicación de las acciones propuestas, se utilizó el Método RIAM (Rapid Impact Assessment Matrix) de PASTAKIA (2002). Para esto, se definieron las componentes a evaluar en el análisis de las agrupaciones de entidades (Figura 1). Estas componentes, se distribuyeron en los campos establecidos por la metodología: Físico Químico (FQ), Biológico Ecológico (BE), Socio Cultural (SC) y Económico Operacional (EO). En el caso particular del presente estudio, la evaluación ambiental se sustentó en la comparación de las actuales agrupaciones, con las nuevas que se forman, a partir de la aplicación de las alternativas propuestas.

La evaluación de las componentes se hizo, a través de la siguiente fórmula:

$$
(a 1) \times(a 2)=a \mathrm{~T} ;(b 1)+(b 2)+(b 3)=b \mathrm{~T} \text { y }(a \mathrm{~T}) \times(b \mathrm{~T})=\mathrm{ES} \text { donde: }
$$

(a1) y (a2): puntuaciones individuales de los criterios relativos a la importancia del impacto (A); (b1) a (b3): puntuaciones individuales de los criterios relativos al valor del impacto (B); 


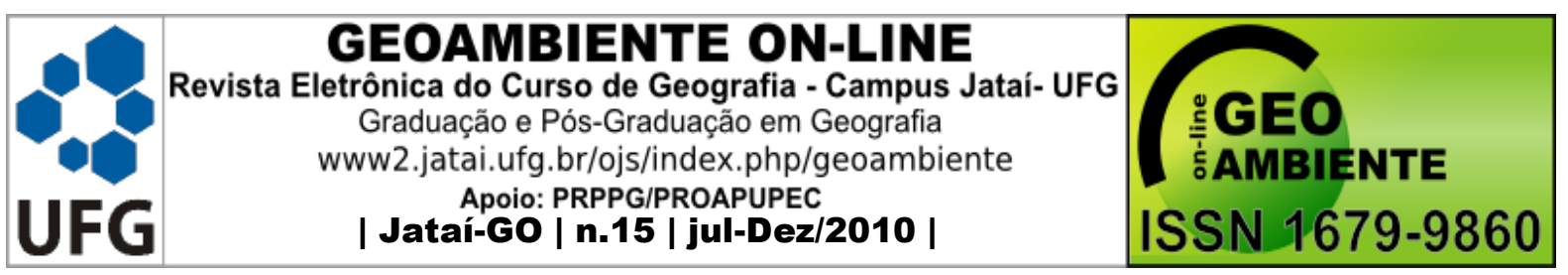

$a \mathrm{~T}$ : resultado de la multiplicación de todas las puntuaciones de $(\mathrm{A}) ; b \mathrm{~T}$ : suma de todas las puntuaciones de (B) y ES: puntuación que evalúa cada componente.

Los criterios de evaluación de cada componente, se ubicaron en clases (Cuadro 1), lo que permitió establecer las comparaciones entre los grupos. En la evaluación, se tomó como valor de la clase, la media de la banda.

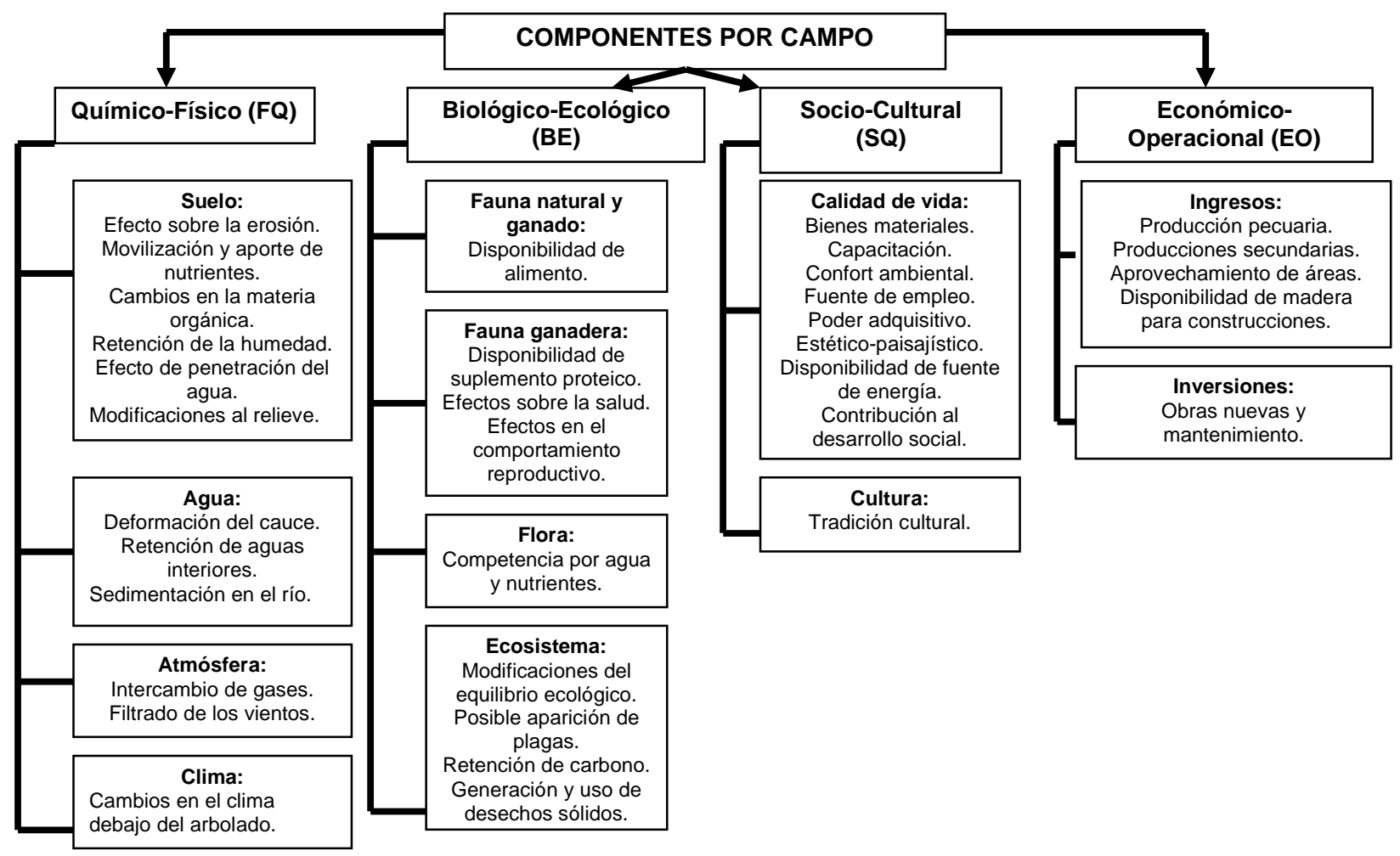

Figura 1 Componentes por campo consideradas en el análisis del impacto ambiental.

Cuadro 1. Bandas de rangos que se agrupan en clases para expresar el comportamiento de los componentes dentro de cada campo.

\begin{tabular}{|cccccccccccc|}
\hline Bandas & -108 & -71 & -35 & -18 & -9 & 0 & 1 & 10 & 19 & 36 & 72 \\
& -72 & -36 & -19 & -10 & -1 & 0 & 9 & 18 & 35 & 71 & 108 \\
\hline Clases & $-\mathrm{E}$ & $-\mathrm{D}$ & $-\mathrm{C}$ & $-\mathrm{B}$ & $-\mathrm{A}$ & $\mathrm{N}$ & $\mathrm{A}$ & $\mathrm{B}$ & $\mathrm{C}$ & $\mathrm{D}$ & $\mathrm{E}$ \\
\hline
\end{tabular}

\section{3.- Resultados y Discusión}

\subsection{Caracterización}

Las variables analizadas mostraron una alta heterogeneidad (Cuadro 2), lo que se pudiera relacionar con las complejidades inherentes a las cuencas hidrográficas, expresadas por Chamichumbi (2007). De esta forma, se encontraron entidades con un área total de 8 


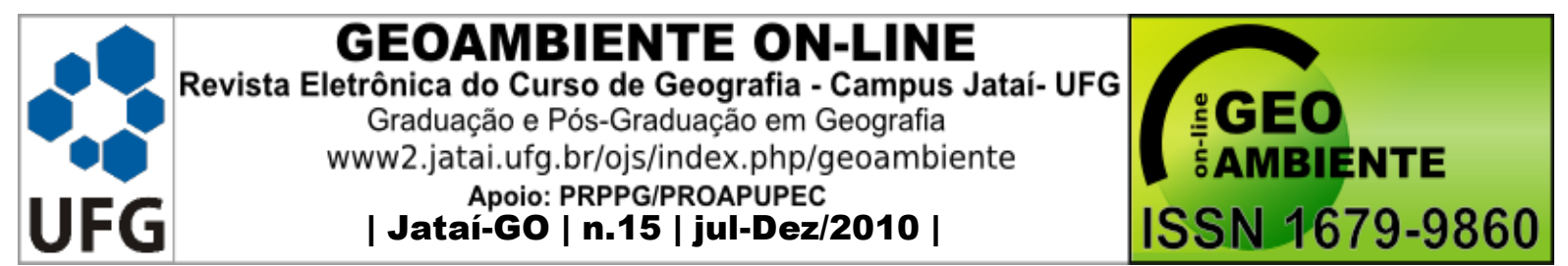

271,4 ha y otras con sólo 53,7 ha $(\mathrm{CV}=95 \%)$. Las producciones de leche en el año indican la existencia de entidades muy productivas con $1425,7 \mathrm{Ml}$ y otras que registran bajas producciones 9,6 $\mathrm{Ml}(\mathrm{CV}=112,9 \%)$. El número de vacas totales se mueve dentro de un intervalo de 1793 y 13 animales como promedio $(\mathrm{CV}=86,6 \%)$ y las variaciones en relación al uso de la tierra son marcadas (áreas de pastos naturales, artificiales, forrajes, con infestaciones de plantas indeseables, (Cuadro 2). Este comportamiento indica la necesidad de agrupar las entidades, buscando similitud entre las mismas, con el fin de lograr una mayor precisión a la hora de planificar y jerarquizar las acciones de mitigación, de acuerdo con los criterios expresados por ARZUBI Y COSTA (2002) y GUEVARA (2005).

Los recorridos de campo, así como la información estadística (Cuadro 1), derivaron la identificación de las principales acciones que desarrolla la actividad, que ocasionan impactos ambientales.

La alta presencia de áreas inutilizadas por el Marabú (42\%) que ha modificado la vegetación original (pastizales), ofrece el criterio de un manejo inadecuado (Cuadro 2). En los recorridos de campo, se pudo constatar además, la ausencia de cuartones para el establecimiento de la rotación, el sobrepastoreo, los elevados tiempos de ocupación y la competencia de los pastos, componentes fundamentales de la flora, con plantas indeseables que invaden los potreros, entre otros factores que afectan la estabilidad y la calidad de la hierba (SENRA, 1993; REYES, 2003).

Lo anterior, unido a las escasas áreas de pastos y forrajes de calidad (14,5\% del área total) y la inestabilidad observada de fuentes alternativas de alimentación, demuestran que no hay garantía de los requerimientos básicos del ganado. Esta situación se agudiza en el período de escasas precipitaciones, todo lo cual perjudica directamente a los animales (SENRA, 2001) y pone de manifiesto que la nutrición merece atención.

El desaprovechamiento del área total disponible para el desarrollo de la actividad económica $\left(0,38\right.$ vacas.ha $\left.^{-1}\right)$, también denota un manejo cuestionable.

La existencia de una cantidad apreciable de estructuras para el abasto de agua, en contraposición con la declaración de insuficiencias en el abasto de la misma (Cuadro 2) invita a la revisión del uso responsable de dicho recurso.

La tala de árboles es una actividad que se vincula en gran medida con el desarrollo de la ganadería (IBRAHIM y MORA-DELGADO, 2003). En la cuenca hidrográfica del río San Pedro, esta acción ya no constituye una práctica sistemática, pero los efectos de la misma en 


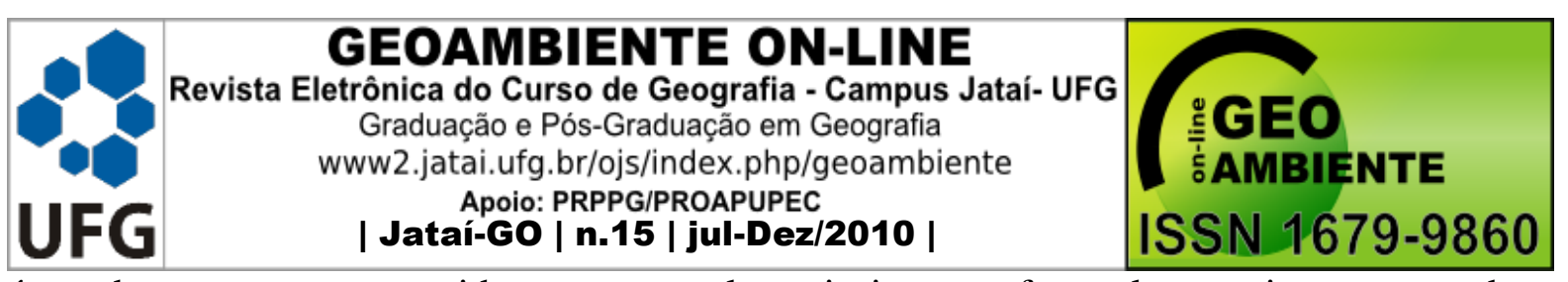

áreas de pastos son muy evidentes, aun, en los paisajes que ofrecen los ecosistemas ganaderos de la cuenca.

La contaminación de aguas interiores se localiza principalmente en el matadero sanitario de la Empresa Pecuaria Triángulo 5, el cual vierte sus residuales sin tratar al medio y los pozos de la zona se encuentran contaminados. Situación análoga pudiera ocurrir en "El Aguacatal" de la Empresa Pecuaria Triángulo 1, donde los taludes se encuentran sucios y se corre el riesgo de filtración de los residuales.

La contaminación de aguas superficiales se manifiesta principalmente en aquellas entidades que vierten sus residuales directamente a los cuerpos de agua, y también en la construcción de tranques dedicados al abasto de agua para el ganado, donde los animales se introducen $\mathrm{y}$ vierten sus deyecciones directamente contaminando los acuatorios y trasmitiendo enfermedades (FAO, 2005).

Cuadro 2. Comportamiento de las entidades ganaderas a través de las variables estudiadas

\begin{tabular}{|cccccc|}
\hline Variable & Media & DS & Variable & Media & DS \\
\hline Área total(ha) & 1656 & 1571,1 & Producción de leche por obrero (Ml) & 5,9 & 10,88 \\
\hline Área con Marabú (ha) & 690 & 1215,9 & Muertes (no) & 140 & 139,2 \\
\hline Pastos cultivados (ha) & 240 & 375,1 & Gastos de salario (M\$) & 431 & 315,8 \\
\hline Pastos naturales (ha) & 948 & 836,7 & Gastos totales (M\$) & 738 & 469 \\
\hline Especies arbóreas (no) & 31 & 15,2 & Gastos por peso(\$) & 1 & 0,3 \\
\hline Pozos (no) & 16 & 12,3 & Total de trabajadores (no) & 87 & 43,5 \\
\hline Tranques (no) & 9 & 11 & Obreros (no) & 62 & 30,2 \\
\hline Molinos (no) & 6 & 5 & Técnicos (no) & 7 & 11 \\
\hline Micropresas (no) & 0,5 & 0,85 & Trabajadores de Servicios (no) & 10,8 \\
\hline Nivel de satisfacción de & 87 & 17,1 & Producción de leche por ha (Ml) & 199,4 & 144,82 \\
agua (\%) & & & Producción de leche por Total de & 4,3 & 8,52 \\
\hline Vacas (no) & 623 & 539,3 & trabajadores (Ml) & 546 & 270,7 \\
\hline Producción de leche (Ml) & 312 & 352,6 & Producción de leche por vaca (l) & 546,3 \\
\hline Precio de la leche (\$) & 1 & 0,1 & Gastos de salario por ha (M\$) & 0,4 & 0,33 \\
\hline Nacimientos (no) & 389 & 320,1 & Gastos totales por ha (M\$) & 0,7 & 0.56 \\
\hline
\end{tabular}

La construcción de tranques y microembalses en las entidades de la cuenca, son ejemplos de la ejecución de obras sin microlocalización, ni autorización ambiental. Esto, de conjunto con la deforestación y el manejo irracional de los pastos, han contribuido a la transformación del paisaje rural.

El uso inadecuado de productos químicos se refiere básicamente al almacenamiento de pesticidas relativo a la Empresa de Cultivos Varios de Camagüey, lo cual ha sido objeto de 


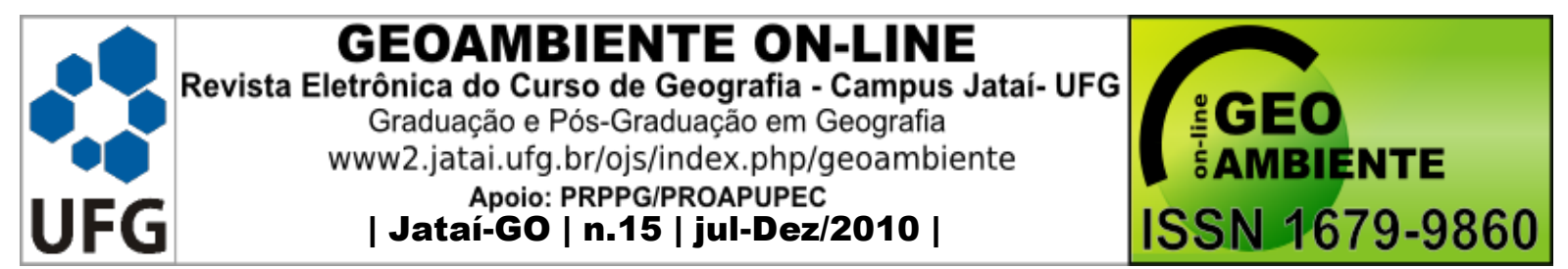

señalamientos por el Grupo de Inspección del Ministerio de Ciencia, Tecnología y Medio Ambiente en Camagüey (ANÓN., 2001).

El ganado que se ubica en áreas de pastos próximas a caminos y carreteras donde las cercas se encuentran en mal estado, es protagonista de posibles accidentes. También los camiones cargados con ganado y productos agrícolas mal asegurados, constituyen elementos generadores de estos impactos.

El transporte que se utiliza para el traslado de ganado y sus producciones, genera consumo de combustible y emisión de gases que contaminan la atmósfera (MURGUEITIO, 2003). También, el ganado tributa a la atmósfera emisiones de metano ocasionadas por la fermentación entérica.

Como resultado del trabajo de análisis desarrollado por los expertos, se definieron como acciones que generan impactos las siguientes: A: tala; B: manejo de pastizales establecidos; C: alimentación animal; D: trabajo de selección genética; E: construcción de tranques para el abasto de agua a la ganadería; F: funcionamiento de mataderos sanitarios; G: uso de productos químicos; H: mantenimiento de viales; I: mantenimiento de instalaciones; J: hurto y sacrificio ilegal de ganado mayor; K: traslado de producciones; L: desarrollo de comunidades rurales; M: construcción de instalaciones pecuarias y otras; N: producción pecuaria; $\tilde{\mathrm{N}}$ : siembra de especies arbóreas y pratenses para la alimentación animal y O: limpieza de potreros.

Los impactos identificados y su grado de importancia sobre cada componente y subsistema, se puede apreciar en la matriz de evaluación (Cuadro 3).

En relación con las componentes del Subsistema Naturaleza, se constató que el ganado lechero es el que recibe una mayor afectación, producto de las acciones antrópicas que se realizan en la cuenca, lo que se pone de manifiesto con un indicador de impacto que clasifica como MUY ALTO (- 334). Este resultado es consistente con los principios básicos y fundamentos agroecológicos, los cuales analizan la explotación agropecuaria como un sistema (MONZOTE, 2005) dentro del cual, el ganado y su producción es el resultado final, donde se concretan todas las acciones desarrolladas. Por lo que las afectaciones que se identifican sobre esta componente, constituyen según DE LA COLINA (2005) y RODRÍGUEZ (2005), una consecuencia del manejo inadecuado de los recursos disponible. 


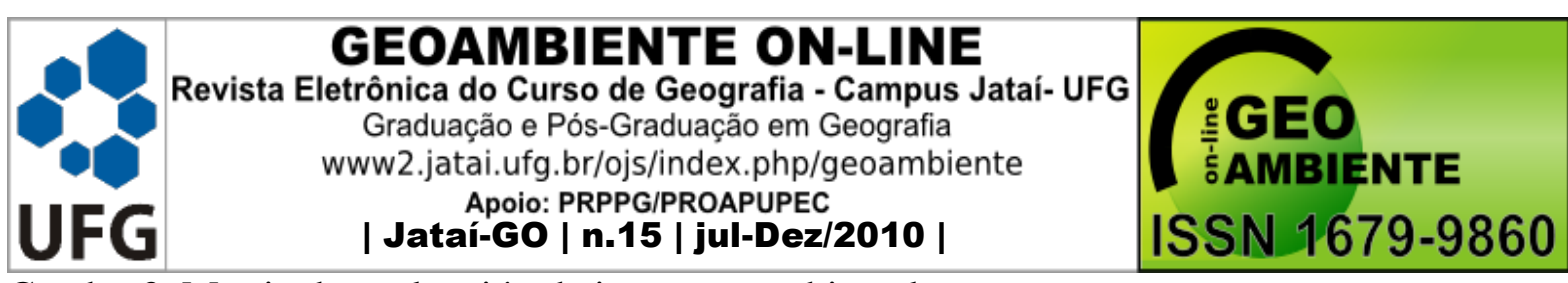

Cuadro 3. Matriz de evaluación de impactos ambientales

\begin{tabular}{|c|c|c|c|c|c|c|c|c|c|c|c|c|}
\hline \multirow[t]{2}{*}{ Impacto } & \multicolumn{8}{|c|}{ Subsistema Naturaleza } & \multicolumn{4}{|c|}{ Subsistema Socioeconomía } \\
\hline & I & II & III & IV & $\mathbf{V}$ & VI & VII & A & VIII & IX, & B & $\mathbf{C}$ \\
\hline 1 & & -17 & -16 & -31 & -61 & -57 & -37 & -219 & & -16 & -16 & -235 \\
\hline 2 & & -17 & -20 & -26 & -31 & -31 & -29 & -154 & & -15 & -15 & -169 \\
\hline 3 & & & & -15 & -15 & -29 & -15 & -74 & & -12 & -12 & -86 \\
\hline 4 & & & & 14 & 13 & 13 & -20 & 20 & & -36 & -36 & -16 \\
\hline 5 & -13 & & -12 & -63 & -39 & -39 & -37 & -203 & & -39 & -39 & -242 \\
\hline 6 & -10 & & -26 & -33 & -21 & -21 & -13 & -124 & & -10 & -10 & -134 \\
\hline 7 & & -10 & & & & & & -10 & & & & -10 \\
\hline 8 & & & & & & -27 & & -27 & & -23 & -23 & -50 \\
\hline 9 & & & & & & -43 & & -43 & & -25 & -25 & -68 \\
\hline 10 & & & & & & -15 & & -15 & & -11 & -11 & -26 \\
\hline 11 & & & & & & -12 & & -12 & & -10 & -10 & -22 \\
\hline 12 & & & & & -30 & -30 & & -60 & & -20 & -20 & -80 \\
\hline 13 & & & & & & -18 & & -18 & & & & -18 \\
\hline 14 & & & -12 & & & & & -12 & -12 & & -12 & -24 \\
\hline 15 & & & & & & & & & -13 & & -13 & -13 \\
\hline 16 & & & & & & & -42 & -42 & & & & -42 \\
\hline 17 & & & & & & -28 & & -28 & & -35 & -35 & \begin{tabular}{|l|}
-63 \\
\end{tabular} \\
\hline 18 & & & & 27 & -28 & -29 & -28 & -58 & & -23 & -23 & -81 \\
\hline 19 & -12 & & & -19 & & 31 & & 0 & & 29 & 29 & 29 \\
\hline 20 & & & & & & -12 & & -12 & & & & -12 \\
\hline 21 & & & -22 & & & -12 & & -34 & & & & -34 \\
\hline 22 & -10 & & -22 & & -12 & & & -44 & & & & -44 \\
\hline 23 & & & & & & & & & -11 & & -11 & -11 \\
\hline 24 & & & & & & & & & -12 & -15 & -27 & -27 \\
\hline 25 & & & & & & -11 & & -11 & -11 & & -11 & -22 \\
\hline 26 & & & & & & & & & 47 & 30 & 77 & 77 \\
\hline 27 & & & & & & & & & 30 & 47 & 77 & 77 \\
\hline 28 & & 18 & 18 & 18 & 18 & 18 & 18 & 108 & 18 & 18 & 36 & 144 \\
\hline 29 & & & & 18 & 18 & 18 & 18 & 72 & 18 & 18 & 36 & 108 \\
\hline Total & -45 & -26 & -112 & -110 & -188 & -334 & -185 & -1000 & 54 & -148 & -94 & -1094 \\
\hline
\end{tabular}

1: deforestación; 2: afectaciones a la flora y a la vegetación; 3: destrucción de hábitat y nichos ecológicos; 4: cambio de uso de suelo; 5: erosión; 6: afectación al drenaje; 7: afectaciones a la atmósfera; 8: desnutrición animal; 9: comportamiento de indicadores reproductivos; 10: incidencia de enfermedades; 11: mortalidad; 12: deterioro del potencial genético; 13: contaminación de aguas superficiales; 14: contaminación de aguas interiores; 15: generación de desechos sólidos; 16: transformación del paisaje; 17: baja productividad; 18: introducción de vegetación indeseable; 19: modificación del relieve; 20: aumento de sedimentos en el cause del río; 21: variación del gasto ecológico del río; 22: deformación de causes; 23: presencia de vectores; 24: pérdidas económicas; 25: accidentes del tránsito; 26: generación de empleos; 27: contribución al desarrollo económico y social de la región; 28: nuevas fuentes de abasto de alimento para el ganado y 29: recuperación de áreas para el pastoreo; A: indicador del impacto en el Subsistema Naturaleza; B: indicador del impacto en el Subsistema Socioeconomía; C: indicador general del impacto.

En una segunda y tercera posición quedan las afectaciones ocasionadas a la flora y la vegetación y al paisaje, ambas con un indicador ALTO (-188 y -185, respectivamente). 


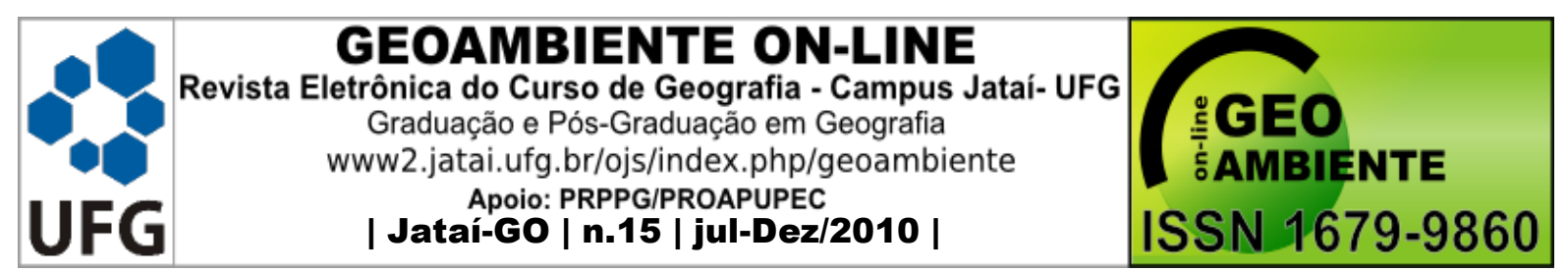

La componente suelo por su parte, clasificó con un nivel de afectación BAJO (-110), sin embargo, el suelo es la base del sistema y toda afectación que reciba por mínima que resulte, repercute en el resto de los elementos en mayor o menor grado. Además, estas afectaciones pueden desencadenar procesos que a mediano plazo, resulten irreversibles o cuya solución sea extraordinariamente costosa.

En el caso de las componentes del Subsistema de Socioeconomía, la más impactada resultó ser la producción de recursos materiales, que reportó in indicador de impacto MEDIO $(-148)$

En el Subsistema Naturaleza completo, los impactos que más incidieron de forma negativa y en orden de importancia fueron: la deforestación, la erosión de los suelos, las afectaciones a la flora y la vegetación y las afectaciones al drenaje. Por otra parte, en todo el Subsistema de Socioeconomía los impactos negativos de mayor importantes fueron: la erosión de los suelos, el cambio de uso de la tierra y la baja productividad alcanzada.

\subsection{Clasificación}

El análisis arrojó que el agrupamiento de las entidades fue diferente en las dos partes de la cuenca: tres grupos de entidades en la parte alta y cuatro grupos en la parte baja.

El comportamiento de los principales recursos en la PA (Cuadro 4) mostró que el Grupo I tiene las 9 entidades más pequeñas (cinco cooperativas con usufructo de la tierra (UBPC), tres cooperativas dueñas de la tierra (CPA) y una Granja Estatal). Este grupo realiza un control adecuado de plantas indeseables como el Marabú (Dichrostachys cinerea (L.) Wight \& Arn.) con un 9, 3\% de infestación en su área total; sin embargo, disponen de poco pasto cultivado $(13,4 \%)$, son pobres en diversidad arbóreas y tienen un bajo aprovechamiento del área total $\left(0,38\right.$ vacas.ha $\left.^{-1}\right)$. La disponibilidad de agua es adecuada en estas entidades, pero tienen poca infraestructura para garantizar el suministro del recurso. No cuentan con un buen balance de su fuerza laboral $\left(0,24\right.$ trabajadores.$\left.h^{-1}\right)$, con sólo el $5 \%$ de técnicos de nivel medio y $6 \%$ trabajadores en los servicios (Cuadro 4). 


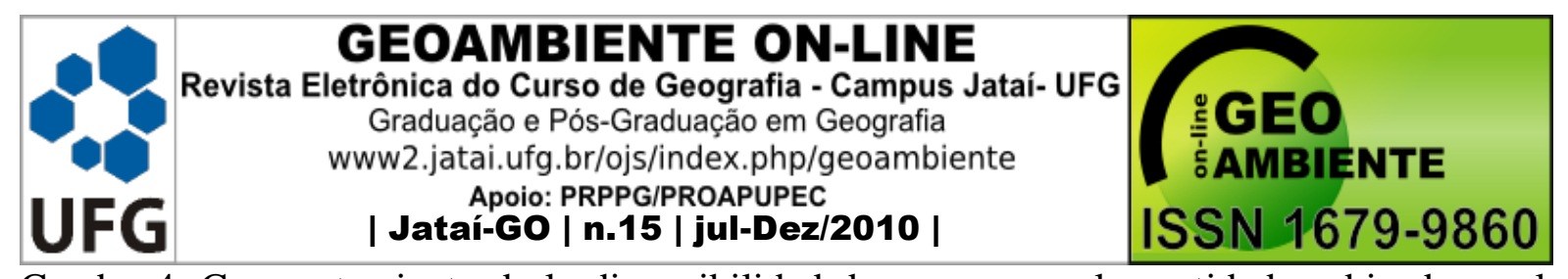

Cuadro 4. Comportamiento de la disponibilidad de recursos en las entidades ubicadas en la parte alta de la cuenca del río San Pedro.

\begin{tabular}{|crrrrrr|}
\hline \multirow{2}{*}{ Variables } & \multicolumn{2}{c}{ Grupo I } & \multicolumn{2}{c}{ Grupo II } & \multicolumn{2}{c|}{ Grupo III } \\
\cline { 2 - 7 } & Media & \multicolumn{1}{c}{ DS } & \multicolumn{1}{c|}{ DS } & Media & \multicolumn{1}{c|}{ DS } \\
\hline AREAT & 287,5 & 207,68 & 1974,9 & 568,07 & 2533,4 & 167,43 \\
\hline ÁREAM & 26,9 & 42,05 & 622,5 & 453,36 & 275,9 & 390,28 \\
\hline PASTOSA & 38,6 & 59,86 & 389,3 & 186,32 & 872,5 & 1233,90 \\
\hline PASTON & 223,1 & 184,93 & 1005,3 & 578,15 & 1875,9 & 1097,28 \\
\hline NOARB & 17,9 & 14,73 & 44,4 & 7,44 & 46,5 & 7,78 \\
\hline TT & 69,3 & 36,83 & 126,2 & 28,16 & 86,5 & 95,46 \\
\hline TEC & 3,2 & 2,63 & 10,7 & 4,00 & 8,5 & 10,61 \\
\hline SERV & 3,8 & 3,48 & 20,4 & 12,72 & 18,5 & 24,75 \\
\hline POZO & 7,1 & 12,47 & 25,3 & 6,16 & 14,5 & 14,85 \\
\hline MOLINO & 1,4 & 1,42 & 8,5 & 5,62 & 3,5 & 3,54 \\
\hline TRANQUE & 1,8 & 1,56 & 20,7 & 11,56 & 6,5 & 4,95 \\
\hline MICRO & 0,0 & 0,00 & 0,5 & 0,71 & 1,0 & 1,42 \\
\hline NSAT & 91,7 & 17,68 & 72,5 & 18,45 & 100,0 & 0,00 \\
\hline VACAS & 108,4 & 94,588 & 982,22 & 355,099 & 1299,60 & 115,117 \\
\hline
\end{tabular}

El Grupo II tiene 10 entidades (ocho UBPC y dos Granjas Estatales) con una extensión media. Es de los tres grupos, el que presenta una mayor infestación de especies indeseables en sus pastizales (31,5\% del área total). La cobertura de pastos cultivados es superior a la del Grupo I (19,7\% del área total), pero no a la del Grupo III. De forma similar se comporta el aprovechamiento del área total (0,50 vacas.ha $\left.{ }^{-1}\right)$ (Cuadro 4). Es el Grupo que reporta la mayor cantidad de pozos, molinos y tranques; sin embargo, el nivel de satisfacción de abasto de agua es el más bajo, lo que se pudiera relacionar con una ubicación inadecuada de estas obras (Cuadro 4).

Con relación a la fuerza laboral, tienen el mayor porcentaje de trabajadores por unidad de área $(6,4 \%)$ y más técnicos y trabajadores de servicios $(8,5 \%$ y $16,6 \%$, respectivamente).

El Grupo III lo forma sólo dos entidades (una UBPC y una CPA), ambas con buen desempeño productivo, disponen de un área total que representa el 55,8\% de la PA con un control adecuado de plantas indeseables (10,9\% del área total). Los pastos cultivados cubren alrededor del 34,4\% y de los tres grupos, es el de mejor diversidad arbórea, lo que presupone la mejor base alimenticia. Tiene un adecuado aprovechamiento del área total para 0,69 vacas.ha ${ }^{-1}$ y reportan cubiertas las necesidades de agua (Cuadro 4). Poseen el menor número de trabajadores por unidad de área $(0,03)$ y la mejor fuerza calificada $(10 \%$ de técnicos), lo que indica una mayor eficiencia en el empleo de los recursos humanos. 


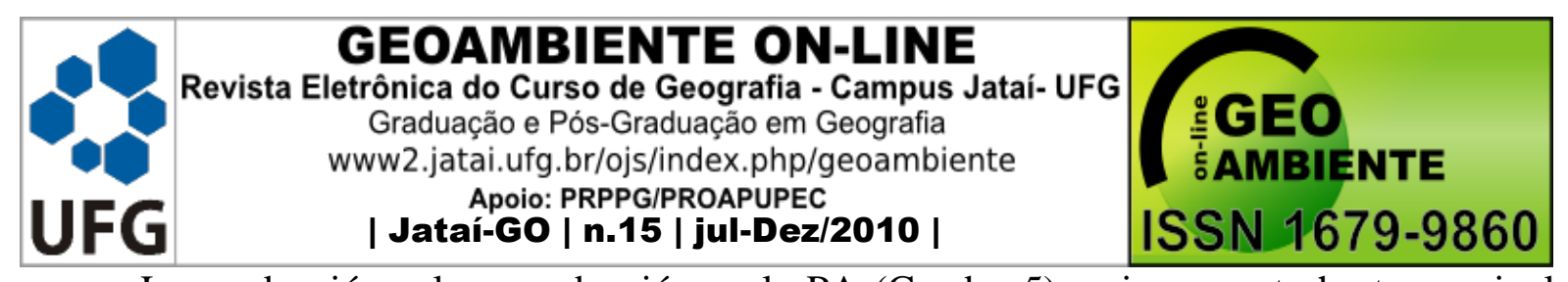

La producción y la reproducción en la PA (Cuadro 5) se incrementa hasta un nivel, entre el Grupo I y el Grupo III; este último con un alto nivel de operaciones, con el $70 \%$ del total de la leche que se recoge durante un año en toda la PA y las entidades que más leche acopian en el país (Cuadro 5).

Cuadro 5. Comportamiento de los indicadores productivos y reproductivos de las entidades ubicadas en la parte alta de la cuenca del río San Pedro.

\begin{tabular}{|c|c|c|c|c|c|c|}
\hline \multirow[b]{2}{*}{ Variables } & \multicolumn{2}{|c|}{ Grupo I } & \multicolumn{2}{|c|}{ Grupo II } & \multicolumn{2}{|c|}{ Grupo III } \\
\hline & Media & DS & Media & DS & Media & DS \\
\hline PLECHE & 56,3 & 45,06 & 500,3 & 151,87 & 1234,6 & 270,34 \\
\hline NAC & 49,4 & 55,56 & 586,1 & 180,89 & 908,5 & 102,53 \\
\hline MUERTE & 32,8 & 59,42 & 222,7 & 158,23 & 174,7 & 24,75 \\
\hline GASTOSA & 154,6 & 158,20 & 5653,3 & 172,73 & 835,4 & 839,11 \\
\hline GASTOT & 339,7 & 348,03 & 1028,9 & 258,27 & 1492,9 & 683,36 \\
\hline GASTOPP & 1,1 & 0,20 & 1,1 & 0,16 & 0,7 & 0,06 \\
\hline PLXHA & 188,9 & 82,95 & 263,9 & 89,89 & 491,9 & 139,22 \\
\hline NACXHA & 0,1 & 0,10 & 0,3 & 0,09 & 0,4 & 0,06 \\
\hline $\begin{array}{c}\text { GASALX } \\
\text { HA }\end{array}$ & 0,7 & 0,52 & 0,3 & 0,08 & 0,3 & 0,35 \\
\hline GASTOTXHA & 1,3 & 0,88 & 0,6 & 0,19 & 0,6 & 0,31 \\
\hline PLXVACA & 0,6 & 0,16 & 0,5 & 0,15 & 0,9 & 0,12 \\
\hline PLXTT & 1,0 & 1,08 & 4,0 & 0,92 & 32,1 & 32,28 \\
\hline PLXOBRE & 1,3 & 1,30 & 5,9 & 1,28 & 42,3 & 38,57 \\
\hline NACXVAC & 0,4 & 0,23 & 0,6 & 0,11 & 0,7 & 0,02 \\
\hline
\end{tabular}

La PLXHA en el Grupo III es 2,6 y 1,9 veces mayor, que la que se produce en los Grupos I y II, respectivamente y los NACXVACA también resultan favorables al Grupo III. La relación que se establece entre el GASTOT y el GASTOS es de 45,5; 54,9 y 56\% para los Grupos I, II y III, respectivamente y es consecuente con las eficiencias productiva y reproductiva de los grupos. El GASTOPP en el Grupo III, se aproxima al reportado por Iglesias (2006) en explotaciones bajo sistemas silvopastoriles.

En la PB la disponibilidad de recursos ofrece perspectivas menores para el desarrollo de la ganadería bovina (Cuadro 6). El Grupo II incluye las cuatro entidades mayores (Granjas Estatales), que en su conjunto ocupan el 43,8\% del área total. Le sigue el Grupo IV también con cuatro entidades (UBPC) y el 29,2\% (11 323,77 ha), luego el Grupo I con nueve entidades (dos UBPC, tres CPA y cuatro Granjas Estatales) con un área total de 953,5 ha, para un $22,1 \%$ y, finalmente, el Grupo III con dos entidades (Granjas Estatales) que ocupan el $4,9 \%$ de la localidad. 


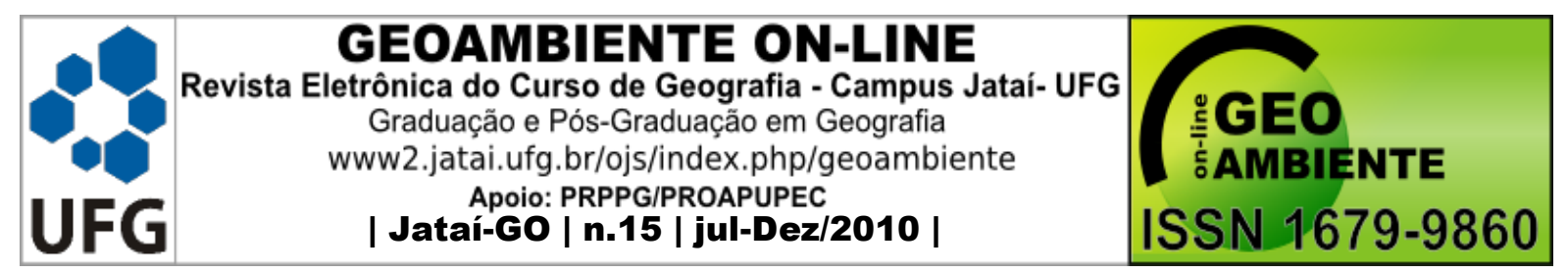

Cuadro 6. Comportamiento de la disponibilidad de recursos de las entidades ubicadas en la parte baja de la cuenca del río San Pedro.

\begin{tabular}{|crrrrrrrr|}
\hline Variables & \multicolumn{2}{c}{ Grupo I } & \multicolumn{2}{c}{ Grupo II } & \multicolumn{2}{c|}{ Grupo III } & \multicolumn{2}{c|}{ Grupo IV } \\
\cline { 2 - 9 } & Media & \multicolumn{1}{c}{ DS } & Media & DS & Media & DS & Media & DS \\
\hline SUELO E & 4,0 & 0,00 & 4,0 & 0,00 & 4,0 & 0,00 & 3,5 & 0,58 \\
\hline AREAT & 953,5 & 648,34 & 4259,6 & 3028,79 & 949,4 & 490,31 & 2830,9 & 1207,87 \\
\hline AREAM & 321,7 & 362,63 & 3244,4 & 2704,65 & 605,8 & 71,57 & 873,9 & 524,55 \\
\hline PASTOSA & 0,4 & 1,31 & 547,6 & 426,88 & 169,0 & 56,57 & 267,9 & 508,22 \\
\hline PASTON & 723,0 & 601,41 & 1400,9 & 750,79 & 473,0 & 55,15 & 2259,5 & 1023,17 \\
\hline FORR & 14,8 & 20,94 & 16,7 & 7,77 & 24,2 & 4,10 & 134,6 & 53,82 \\
\hline TT & 47,1 & 26,21 & 107,2 & 19,24 & 74,5 & 16,26 & 108,2 & 35,16 \\
\hline OBRE & 35,9 & 20,35 & 78,2 & 11,64 & 48,0 & 0,00 & 73,7 & 30,15 \\
\hline TEC & 2,7 & 2,24 & 9,2 & 3,50 & 10,5 & 9,19 & 10,2 & 4,72 \\
\hline SERV & 3,6 & 2,69 & 9,7 & 6,39 & 8,0 & 7,07 & 14,5 & 6,45 \\
\hline DIRIG & 3,4 & 2,69 & 8,0 & 1,41 & 7,0 & 1,41 & 7,0 & 1,83 \\
\hline POZO & 7,3 & 9,00 & 20,0 & 13,46 & 21,5 & 4,95 & 22,2 & 10,05 \\
\hline MOLINO & 2,9 & 1,69 & 10,0 & 7,83 & 9,0 & 1,41 & 8,0 & 3,27 \\
\hline TRANQUE & 1,0 & 1,80 & 16,5 & 11,21 & 8,0 & 2,83 & 8,0 & 11,52 \\
\hline VACAS & 262,7 & 189,60 & 936,4 & 648,60 & 250,7 & 288,07 & 1228,8 & 445,55 \\
\hline
\end{tabular}

El control de las plantas indeseables es un problema en la PB. El Grupo IV es el que reporta menores áreas afectadas con el 30,9\% del total disponible, seguido por el Grupo I con un $34 \%$, el Grupo III con el 63,8\% y el Grupo II con el 76,2\%.

El área de pastos cultivados es favorable en las entidades del Grupo IV (24\% de su área total), mientras que los Grupos III y II tienen el 18 y el 13\%, respectivamente. El Grupo I, prácticamente, no dispone de pastos cultivados. De forma análoga se comporta la tenencia de forrajes con el 5; 2,5; 1,6 y 0,4\% para los Grupos IV; III; I y II, respectivamente. En sentido general, el Grupo IV es el que manifiesta un mejor balance de sus áreas para la garantía del alimento básico.

Cuando se evalúa el total de trabajadores con relación al área total de cada grupo, el indicador más alto lo manifiesta el Grupo III (7,9\%), seguido por el Grupo I (4,9\%), el Grupo IV $(3,8 \%)$ y finalmente el Grupo II $(2,5 \%)$.

El Grupo I tiene un número mayor de obreros $(76,2 \%)$ y está por encima de los que poseen mayores áreas (Grupo II, 73\% y Grupo IV, 68\%), mientras que el Grupo III reporta el menor registro con un $64 \%$ de obreros. El porcentaje de técnicos fue del 14,1; 9,5; 8,7 y 5,7\% para los Grupos III; IV; II y I, respectivamente. 


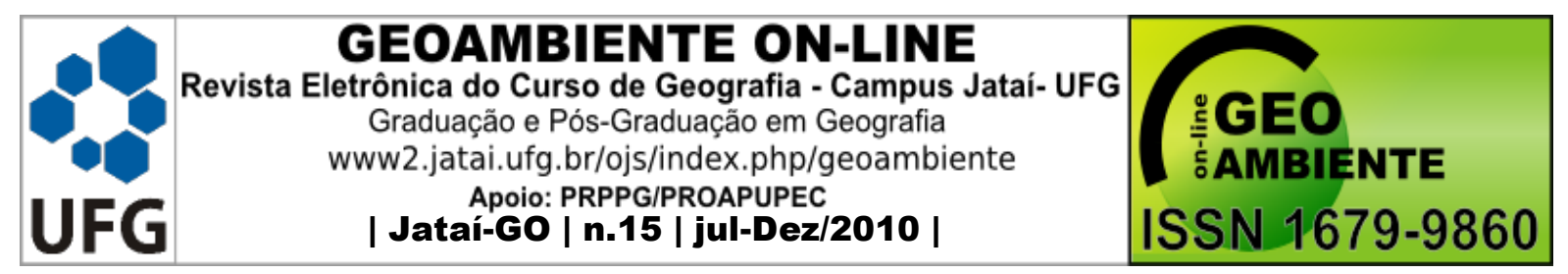

Los trabajadores dedicados al servicio están más representados en las entidades que integran los Grupos IV y III (13,4\% y $10,7 \%$, respectivamente) y menos en las entidades de los Grupos II y I (9,1\% y 7,6\% respectivamente).

Finalmente, los dirigentes representan el $10,7 \%$ de la fuerza laboral de las entidades del Grupo III, seguido por los Grupos II, 1 y el IV (7,5\%; 7,2\% y 6,5\%, respectivamente).

En la PB, el nivel de satisfacción, en relación al abasto de agua para el ganado, es aceptable para todas las entidades; sin embargo, las que se agrupan en IV y III poseen la mejor estructura para el abasto.

El aprovechamiento del área para la producción de leche favorece al Grupo IV, el cual cuenta con un indicador de 0,43 vacas.ha $^{-1,}$ seguido por los grupos I; III y II con 0,28; 0,26 y 0,22, respectivamente. Los indicadores productivos y reproductivos en la PB (Cuadro 7) muestran que las entidades del Grupo IV tienen una producción global de leche que supera al resto de los grupos de entidades de esta parte de la cuenca.

Cuadro 7. Comportamiento de las variables relativas a los indicadores productivos y reproductivos de las entidades ubicadas en la parte baja de la cuenca del río San Pedro.

\begin{tabular}{|crrrrrrrr|}
\hline Variables & \multicolumn{2}{c}{ Grupo I } & \multicolumn{2}{c}{ Grupo II } & \multicolumn{2}{c|}{ Grupo III } & \multicolumn{2}{c|}{ Grupo IV } \\
\cline { 2 - 9 } & Media & DS & Media & DS & Media & DS & Media & DS \\
\hline PLECHE & 111,7 & 80,53 & 144,8 & 60,46 & 82,8 & 25,39 & 691,7 & 477,98 \\
\hline NAC & 153,8 & 123,90 & 414,9 & 231,56 & 646,8 & 230,52 & 771,4 & 219,23 \\
\hline MUERTE & 46,9 & 46,06 & 226,8 & 67,55 & 103,1 & 17,39 & 299,9 & 166,04 \\
\hline GASTOSA & 227,2 & 149,44 & 815,4 & 88,06 & 412,5 & 44,53 & 594,9 & 285,97 \\
\hline GASTOT & 397,7 & 236,06 & 1208,8 & 284,03 & 545,6 & 15,13 & 919,2 & 318,92 \\
\hline GASTOPPP & 1,0 & 0,25 & 0,6 & 0,25 & 0,7 & 0,24 & 1,2 & 0,36 \\
\hline PLXHA & 122,7 & 64,79 & 43,9 & 29,34 & 92,6 & 21,09 & 296,8 & 264,65 \\
\hline NACXHA & 0,2 & 0,08 & 0,1 & 0,14 & 0,7 & 0,13 & 0,3 & 0,12 \\
\hline PLXTT & 2,7 & 1,77 & 1,4 & 0,80 & 1,1 & 1,10 & 6,1 & 2,46 \\
\hline PLXOBRE & 3,6 & 2,37 & 1,9 & 0,89 & 1,7 & 1,72 & 9,0 & 2,96 \\
\hline NACXVAC & 0,6 & 0,11 & 0,5 & 0,22 & 6,0 & 6,04 & 0,6 & 0,07 \\
\hline
\end{tabular}

Los nacimientos, como indicador clave del comportamiento reproductivo (BERTOT, 2006), también distinguen al Grupo IV del resto sin embargo; se aprecia que el Grupo III manifiesta un excelente comportamiento de este indicador, por cuanto tiene un menor número de vacas y los mejores nacimientos por hectárea y por vaca.

De acuerdo con GUEVARA (2005) la PLXOBRE es un indicador de gran importancia en relación a la eficiencia productiva. Este indicador en la PB también favorece, ampliamente, a las entidades del Grupo IV. Por otra parte, la relación entre Gastot y Gastosa como 


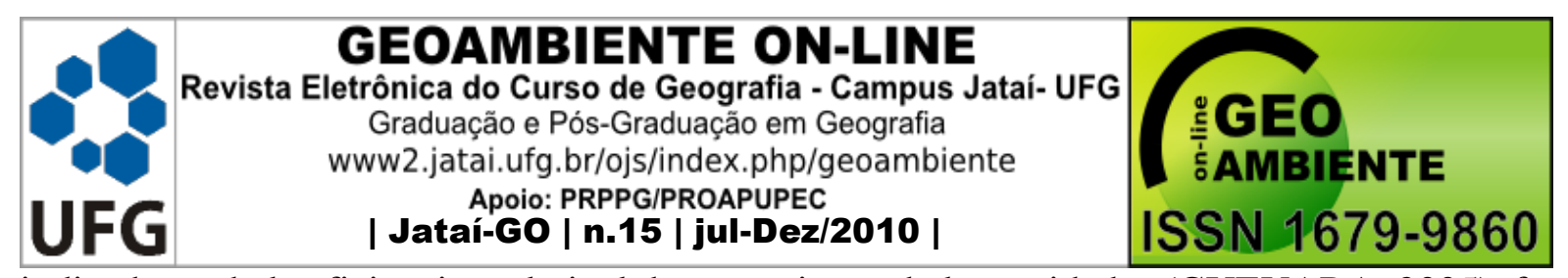

indicadores de la eficiencia y el nivel de operaciones de las entidades (GUEVARA, 2005), fue superior para el Grupo III seguido por el Grupo IV.

En sentido general los Grupos IV y III resultaron similares, en relación a la eficiencia de la gestión económica de sus entidades, por cuanto las del Grupo IV tienen como objeto social la producción de leche y las que integran el Grupo III, el de obtener individuos con valor genético y, en segundo, lugar la producción de leche.

\subsection{Evaluación de impacto ambiental de las alternativas de mitigación que se proponen}

Los resultados alcanzados en la evaluación de las entidades ubicadas en la Parte Alta de la cuenca y sus correspondientes opciones de mitigación muestran que el Grupo I, en el campo FQ, tiene cuatro componentes, en clases, con rangos negativos y ocho en clases positivas, pero de baja puntuación (Cuadro 8). Los componentes negativos, en orden de importancia, fueron: efecto del manejo en la erosión de los suelos, las modificaciones al relieve y causes de afluentes del río y la sedimentación que sufren por el arrastre de suelo.

En el campo EB, seis componentes se encuentran en rangos negativos, con énfasis en los efectos que el sistema ocasiona al comportamiento reproductivo del ganado, la salud y la garantía de alimento para cubrir, en ambas épocas del año, los requerimientos nutricionales. El resto de los componentes negativos, así como los positivos, fueron de baja cuantía.

En el campo SC, sólo la capacitación de los trabajadores se identificó como un componente negativo a considerar, mientras que en el EO el aprovechamiento de las áreas arrojó el valor más negativo, seguido por las bajas producciones secundarias y la disponibilidad de madera para construcciones. El resto de los componentes resultaron positivos.

El Grupo II en el campo FQ manifestó tres componentes negativos; modificaciones al relieve, deformación de causes y la erosión de los suelos. El aporte de sedimentos a los cuerpos de agua se consideró un componente neutro y el resto resultó positivo.

En el campo EB esta agrupación se comportó de manera similar que la anterior, con seis componentes negativos y cuatro positivos; pero los componentes negativos se ubicaron en bandas de rangos más bajos. En el caso del campo SC este grupo de entidades no manifestó ningún componente negativo y tiene a su favor en relación con el Grupo I, que el poder adquisitivo de los trabajadores alcanzó el máximo de puntos. 
Cuadro 8. Distribución de los componentes en las agrupaciones de la parte alta de la cuenca.

\begin{tabular}{|c|c|c|c|c|c|c|c|c|c|c|c|c|}
\hline Media & -90 & -54 & -27 & -14 & -5 & 0 & 5 & 14 & 27 & 54 & 90 & Total \\
\hline Clase & $-E$ & $-D$ & $-\mathrm{C}$ & $-B$ & $-A$ & $\mathrm{~N}$ & A & B & $\mathrm{C}$ & $\mathrm{D}$ & $E$ & \\
\hline \multicolumn{13}{|c|}{ Grupo I } \\
\hline FQ & 0 & 3 & 0 & 1 & 0 & 0 & 7 & 1 & 0 & 0 & 0 & -127 \\
\hline $\mathrm{BE}$ & 1 & 2 & 0 & 2 & 1 & 0 & 1 & 2 & 1 & 0 & 0 & -171 \\
\hline SC & 0 & 0 & 1 & 0 & 0 & 0 & 0 & 4 & 4 & 0 & 0 & 137 \\
\hline EO & 1 & 0 & 2 & 0 & 0 & 0 & 0 & 0 & 2 & 0 & 0 & -90 \\
\hline Total & 2 & 5 & 3 & 3 & 1 & 0 & 8 & 7 & 7 & 0 & 0 & -251 \\
\hline \multicolumn{13}{|c|}{ Grupo I con acciones de mitigación } \\
\hline FQ & 0 & 0 & 0 & 0 & 0 & 2 & 0 & 8 & 1 & 1 & 0 & 193 \\
\hline $\mathrm{BE}$ & 0 & 1 & 0 & 1 & 0 & 0 & 0 & 3 & 2 & 3 & 0 & 192 \\
\hline $\mathrm{SC}$ & 0 & 0 & 0 & 0 & 0 & 0 & 0 & 0 & 3 & 6 & 0 & 405 \\
\hline EO & 0 & 1 & 0 & 0 & 0 & 0 & 0 & 1 & 1 & 2 & 0 & 67 \\
\hline Total & $\mathbf{0}$ & 2 & $\mathbf{0}$ & 1 & $\mathbf{0}$ & 2 & $\mathbf{0}$ & 12 & 7 & 12 & $\mathbf{0}$ & 857 \\
\hline \multicolumn{13}{|c|}{ Grupo II } \\
\hline FQ & 0 & 2 & 1 & 0 & 0 & 1 & 0 & 7 & 1 & 0 & 0 & -10 \\
\hline $\mathrm{BE}$ & 0 & 2 & 2 & 2 & 0 & 0 & 0 & 1 & 2 & 1 & 0 & -68 \\
\hline $\mathrm{SC}$ & 0 & 0 & 0 & 0 & 0 & 0 & 0 & 2 & 4 & 2 & 1 & 334 \\
\hline EO & 0 & 1 & 0 & 0 & 0 & 0 & 0 & 0 & 3 & 1 & 0 & 81 \\
\hline Total & $\mathbf{0}$ & 5 & 3 & 2 & $\mathbf{0}$ & 1 & $\mathbf{0}$ & 10 & 10 & 4 & 1 & 337 \\
\hline \multicolumn{13}{|c|}{ Grupo II con acciones de mitigación } \\
\hline FQ & 0 & 0 & 0 & 0 & 0 & 2 & 0 & 2 & 7 & 1 & 0 & 271 \\
\hline $\mathrm{BE}$ & 0 & 1 & 1 & 0 & 0 & 0 & 0 & 0 & 1 & 6 & 1 & 360 \\
\hline $\mathrm{SC}$ & 0 & 0 & 0 & 0 & 0 & 0 & 0 & 0 & 0 & 5 & 4 & 630 \\
\hline EO & 1 & 0 & 0 & 0 & 0 & 0 & 0 & 0 & 0 & 3 & 1 & 162 \\
\hline Total & 1 & 1 & 1 & $\mathbf{0}$ & $\mathbf{0}$ & 2 & $\mathbf{0}$ & 2 & 8 & 15 & 6 & 1423 \\
\hline \multicolumn{13}{|c|}{ Grupo III } \\
\hline FQ & 0 & 2 & 1 & 0 & 0 & 0 & 0 & 8 & 1 & 0 & 0 & 4 \\
\hline $\mathrm{BE}$ & 0 & 0 & 3 & 3 & 0 & 0 & 0 & 1 & 2 & 1 & 0 & -1 \\
\hline $\mathrm{SC}$ & 0 & 0 & 0 & 0 & 0 & 0 & 0 & 0 & 4 & 4 & 1 & 414 \\
\hline EO & 0 & 0 & 1 & 0 & 0 & 0 & 0 & 0 & 2 & 1 & 1 & 171 \\
\hline Total & $\mathbf{0}$ & 2 & 5 & 3 & $\mathbf{0}$ & $\mathbf{0}$ & $\mathbf{0}$ & 9 & 9 & 6 & 2 & 588 \\
\hline \multicolumn{13}{|c|}{ Grupo III con acciones de mitigación } \\
\hline $\mathrm{FQ}$ & 0 & 0 & 0 & 0 & 0 & 2 & 0 & 2 & 7 & 1 & 0 & 271 \\
\hline $\mathrm{BE}$ & 0 & 1 & 1 & 0 & 0 & 0 & 0 & 0 & 1 & 5 & 2 & 396 \\
\hline $\mathrm{SC}$ & 0 & 0 & 0 & 0 & 0 & 0 & 0 & 0 & 0 & 5 & 4 & 630 \\
\hline EO & 1 & 0 & 0 & 0 & 0 & 0 & 0 & 0 & 0 & 2 & 2 & 198 \\
\hline Total & 1 & 1 & 1 & $\mathbf{0}$ & $\mathbf{0}$ & 2 & $\mathbf{0}$ & 2 & 8 & 13 & 8 & 1495 \\
\hline
\end{tabular}

En el campo EO, solo el aprovechamiento de las áreas se ubicó en una banda con valores negativos; sin embargo, la producción pecuaria de este grupo superó al anterior. En general, el Grupo III es el que manifiesta el balance más favorable. En el campo FQ este grupo mantiene los mismos componentes negativos que el Grupo II. Los componentes negativos incluidos en el campo EB, son los mismos que para el Grupo II y, al igual que en el campo FQ, estos valores son más bajos. El campo SC también se comporta de forma análoga, 


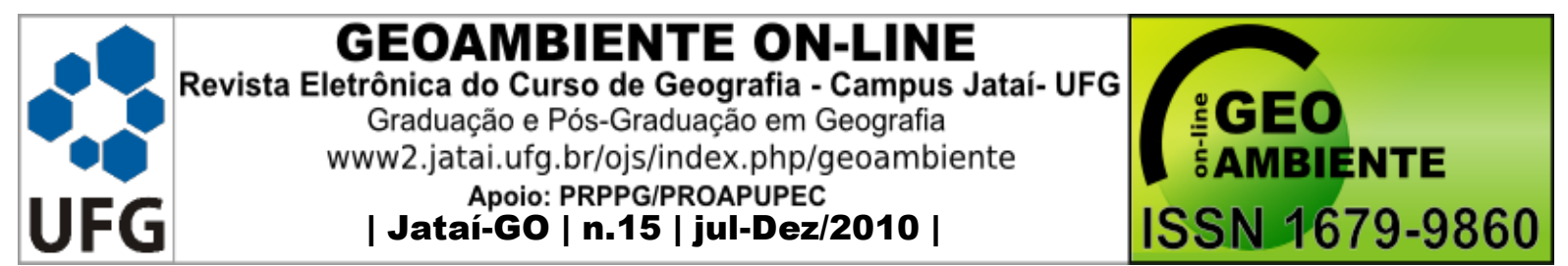

en relación al grupo precedente; pero en el EO el aprovechamiento de las áreas y la producción pecuaria es más favorable.

La comparación cuantitativa entre los efectos en los campos FQ, BE y SC de las agrupaciones actuales y aquellas que incluyen las acciones de mitigación favorecen, a estas últimas, en todos los grupos. En el campo EO se prevé que con la implementación de las acciones, los resultados productivos de las agrupaciones I y II se dupliquen, mientras que para el grupo III se estimó, que el aumento estaría alrededor de una vez más $(1,2)$. Esto se pudiera relacionar con las potencialidades productivas o riqueza de los ecosistemas involucrados, por cuanto y de acuerdo con los criterios desarrollados por ODUM (1987), estos ecosistemas tienden a expresar sus límites y se haría necesario incorporar energía del exterior para lograr mejores resultados.

En el análisis de las entidades ubicadas en la parte baja de la cuenca (Cuadro 9), se pone de manifiesto que en el campo FQ las agrupaciones, una vez implementadas las acciones de mitigación, lograrían pasar de cuatro componentes ubicados en clases negativas (efectos del sistema sobre la erosión del suelo, la presencia de sedimentos en el río, las modificaciones al relieve y las deformaciones al cause); dos a neutras y dos a positivas. En el campo BE se considera que las acciones responderían al déficit de alimentación y suplementación proteica del ganado y, por ello, ambas pasan a clases positivas.

En este campo sin embargo, se prevé la ocurrencia de otros efectos negativos que son la competencia entre por agua y nutrientes del suelo y la posible aparición de plagas, cuestiones asociadas al establecimiento de árboles en pastoreo (FUNES, 2002).

En el caso particular del Grupo V, el cual reporta un alto índice de mortalidad, se consideró que la aplicación de las acciones puede mejorar el indicador, parcialmente, al menos para el tiempo que se evalúa. La capacitación de los trabajadores, en temas directamente vinculados a la aplicación de prácticas que brindan solución a la problemática que hoy persiste en las entidades, es otro aspecto que se prevé resuelto en el campo SC.

En el campo EO las acciones de mitigación lograrían mejorar, considerablemente, el aprovechamiento de las áreas, así como la obtención de producciones secundarias que garanticen nuevos ingresos o reduzcan gastos; sin embargo, aún se precisa continuar trabajando en el mejoramiento de ambos componentes. Las inversiones que se prevén para la garantía futura del desarrollo de la ganadería vacuna en estas entidades, es una componente negativa para esta corta etapa de análisis. 
Cuadro 9. Distribución de los componentes en las agrupaciones de la parte baja de la cuenca.

\begin{tabular}{|c|c|c|c|c|c|c|c|c|c|c|c|c|}
\hline Media & -90 & -54 & -27 & -14 & -5 & $\mathbf{0}$ & 5 & 14 & 27 & 54 & 90 & Total \\
\hline Clase & $-E$ & $-\mathrm{D}$ & $-\mathrm{C}$ & $-B$ & -A & $\mathrm{N}$ & A & B & $\mathrm{C}$ & $\mathrm{D}$ & E & \\
\hline \multicolumn{13}{|c|}{ Grupo IV } \\
\hline $\mathrm{FQ}$ & 0 & 1 & 1 & 2 & 0 & 0 & 7 & 1 & 0 & 0 & 0 & -60 \\
\hline $\mathrm{BE}$ & 0 & 2 & 2 & 1 & 0 & 0 & 1 & 3 & 1 & 0 & 0 & -102 \\
\hline $\mathrm{SC}$ & 0 & 0 & 1 & 0 & 0 & 0 & 0 & 4 & 3 & 1 & 0 & 164 \\
\hline EO & 1 & 1 & 1 & 0 & 0 & 0 & 0 & 0 & 2 & 0 & 0 & -117 \\
\hline Total & 1 & 4 & 5 & 3 & $\mathbf{0}$ & $\mathbf{0}$ & 8 & 8 & 6 & 1 & $\mathbf{0}$ & -115 \\
\hline \multicolumn{13}{|c|}{ Grupo IV con acciones de mitigación } \\
\hline FQ & 0 & 0 & 0 & 0 & 0 & 2 & 0 & 8 & 1 & 1 & 0 & 193 \\
\hline $\mathrm{BE}$ & 0 & 1 & 0 & 0 & 1 & 0 & 0 & 3 & 2 & 3 & 0 & 199 \\
\hline $\mathrm{SC}$ & 0 & 0 & 0 & 0 & 0 & 0 & 0 & 0 & 1 & 7 & 1 & 495 \\
\hline EO & 0 & 1 & 2 & 0 & 0 & 0 & 0 & 0 & 1 & 1 & 0 & -27 \\
\hline Total & $\mathbf{0}$ & 2 & 2 & $\mathbf{0}$ & 1 & 2 & $\mathbf{0}$ & 11 & 5 & 12 & 1 & 860 \\
\hline \multicolumn{13}{|c|}{ Grupo V } \\
\hline FQ & 1 & 3 & 0 & 0 & 0 & 0 & 7 & 1 & 0 & 0 & 0 & -203 \\
\hline $\mathrm{BE}$ & 0 & 1 & 3 & 1 & 0 & 0 & 1 & 3 & 1 & 0 & 0 & -75 \\
\hline $\mathrm{SC}$ & 0 & 0 & 1 & 0 & 0 & 0 & 0 & 4 & 4 & 0 & 0 & 137 \\
\hline EO & 2 & 0 & 1 & 0 & 0 & 0 & 0 & 0 & 2 & 0 & 0 & -153 \\
\hline Total & 3 & 4 & 5 & 1 & $\mathbf{0}$ & $\mathbf{0}$ & 8 & 8 & 7 & 0 & $\mathbf{0}$ & -294 \\
\hline \multicolumn{13}{|c|}{ Grupo V con acciones de mitigación } \\
\hline FQ & 0 & 0 & 0 & 0 & 0 & 2 & 0 & 8 & 1 & 1 & 0 & 193 \\
\hline $\mathrm{BE}$ & 0 & 1 & 1 & 0 & 1 & 0 & 0 & 2 & 1 & 4 & 0 & 185 \\
\hline SC & 0 & 0 & 0 & 0 & 0 & 0 & 0 & 0 & 1 & 8 & 0 & 459 \\
\hline EO & 1 & 2 & 0 & 0 & 0 & 0 & 0 & 0 & 1 & 1 & 0 & -117 \\
\hline Total & 1 & 3 & 1 & $\mathbf{0}$ & 1 & 2 & $\mathbf{0}$ & 10 & 4 & 14 & $\mathbf{0}$ & 720 \\
\hline \multicolumn{13}{|c|}{ Grupo VI } \\
\hline FQ & 1 & 3 & 0 & 0 & 0 & 0 & 0 & 7 & 1 & 0 & 0 & -127 \\
\hline $\mathrm{BE}$ & 0 & 0 & 3 & 1 & 0 & 0 & 1 & 2 & 1 & 1 & 1 & 109 \\
\hline $\mathrm{SC}$ & 0 & 0 & 1 & 0 & 0 & 0 & 0 & 1 & 2 & 5 & 0 & 311 \\
\hline $\mathrm{EO}$ & 0 & 2 & 0 & 0 & 0 & 0 & 0 & 0 & 2 & 1 & 0 & 0 \\
\hline Total & 1 & 5 & 4 & 1 & $\mathbf{0}$ & $\mathbf{0}$ & 1 & 10 & 6 & 7 & 1 & 293 \\
\hline \multicolumn{13}{|c|}{ Grupo VI con acciones de mitigación } \\
\hline FQ & 0 & 0 & 0 & 0 & 0 & 2 & 0 & 4 & 5 & 1 & 0 & 245 \\
\hline $\mathrm{BE}$ & 0 & 1 & 0 & 1 & 0 & 0 & 0 & 1 & 2 & 3 & 2 & 342 \\
\hline $\mathrm{SC}$ & 0 & 0 & 0 & 0 & 0 & 0 & 0 & 0 & 0 & 4 & 5 & 666 \\
\hline EO & 0 & 0 & 2 & 1 & 0 & 0 & 0 & 0 & 0 & 1 & 1 & 76 \\
\hline Total & $\mathbf{0}$ & 1 & 2 & 2 & $\mathbf{0}$ & 2 & $\mathbf{0}$ & 5 & 7 & 9 & 8 & 1329 \\
\hline \multicolumn{13}{|c|}{ Grupo VII } \\
\hline FQ & 0 & 3 & 1 & 0 & 0 & 0 & 0 & 7 & 1 & 0 & 0 & -64 \\
\hline $\mathrm{BE}$ & 0 & 0 & 1 & 3 & 0 & 0 & 1 & 2 & 2 & 1 & 0 & 72 \\
\hline $\mathrm{SC}$ & 0 & 0 & 1 & 0 & 0 & 0 & 0 & 0 & 4 & 4 & 0 & 297 \\
\hline EO & 0 & 1 & 1 & 0 & 0 & 0 & 0 & 0 & 1 & 2 & 0 & 54 \\
\hline Total & $\mathbf{0}$ & 4 & 4 & 3 & $\mathbf{0}$ & $\mathbf{0}$ & 1 & 9 & 8 & 7 & $\mathbf{0}$ & 359 \\
\hline \multicolumn{13}{|c|}{ Grupo VII con acciones de mitigación } \\
\hline FQ & 0 & 0 & 0 & 0 & 0 & 2 & 0 & 4 & 5 & 1 & 0 & 245 \\
\hline $\mathrm{BE}$ & 0 & 1 & 0 & 1 & 0 & 0 & 0 & 0 & 2 & 5 & 1 & 346 \\
\hline $\mathrm{SC}$ & 0 & 0 & 0 & 0 & 0 & 0 & 0 & 0 & 0 & 5 & 4 & 630 \\
\hline EO & 0 & 1 & 0 & 1 & 0 & 0 & 0 & 0 & 1 & 0 & 2 & 139 \\
\hline Total & $\mathbf{0}$ & 2 & $\mathbf{0}$ & 2 & $\mathbf{0}$ & 2 & $\mathbf{0}$ & 4 & 8 & 11 & 7 & 1360 \\
\hline
\end{tabular}




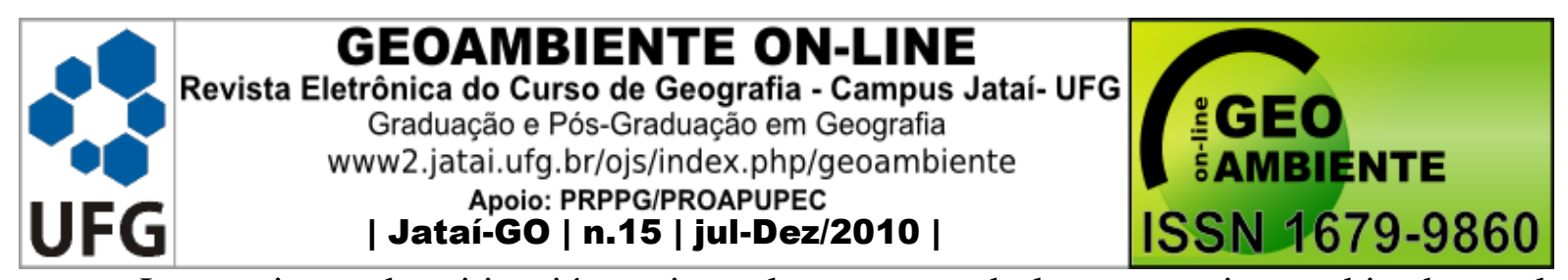

Las opciones de mitigación mejoran la respuesta de las agrupaciones ubicadas en la

Parte Baja de la cuenca (Cuadro 4). Como se observa en la misma, el estado de los ecosistemas ganaderos puede mejorar hasta tres veces, lo que se aprecia, fundamentalmente, en los campos FQ, EB y SQ. Por su parte, en el campo EO, al igual que en la parte alta de la cuenca, las inversiones que se requieren, ocasionarían en la etapa un gasto importante.

\section{4.- Conclusiones y Recomendaciones}

La ganadería bovina ha ocasionado un alto grado de afectación a la cuenca en estudio, con un indicador general de impacto de - 1094 y que hay una elevada heterogeneidad entre las entidades productivas, que amerita de un agrupamiento o clasificación, para planificar y jerarquizar las acciones de mitigación que se deberán acometer.

Las entidades productivas que se dedican al desarrollo de la ganadería bovina, en la cuenca hidrográfica del río San Pedro en Camagüey, Cuba; clasifican en siete grupos bien definidos: tres que se localizan en la parte alta de la cuenca y cuatro en la parte baja o aguas abajo.

Las tecnologías propuestas pueden reconvertir la gestión ambiental de la ganadería bovina en la cuenca hidrográfica bajo estudio, con un saldo muy favorable en relación al desarrollo y la conservación de los ecosistemas existentes. Se recomienda dar continuidad al presente trabajo, con la comprobación y valoración de los resultados que se logren en las entidades productivas, vinculadas con la aplicación de las acciones propuestas.

La secuencia de trabajo utilizada para el ordenamiento de la ganadería bovina en cuenca hidrográfica del río San Pedro en Camagüey, Cuba se pudiera extrapolar a otros escenarios geográficos.

\section{5 - Bibliografía}

ACOSTA, Z.; MARTÍN, G.; PRIMELLES, J. Valoración del impacto ambiental ocasionado por la actividad ganadera en la cuenca del río San Pedro en Camagüey, Cuba. Revista Brasileira de Agroecología 1(1):91-95, 2006.

ANÓN. Informe de Inspección Estatal. Grupo Regulatorio, Unidad de Medio Ambiente del CITMA, Camagüey. Cuba, 5pp, 2001.

ARZUBI, A.; COSTAS, A.M. Escala, eficiencia y productividad en sistemas lecheros de 


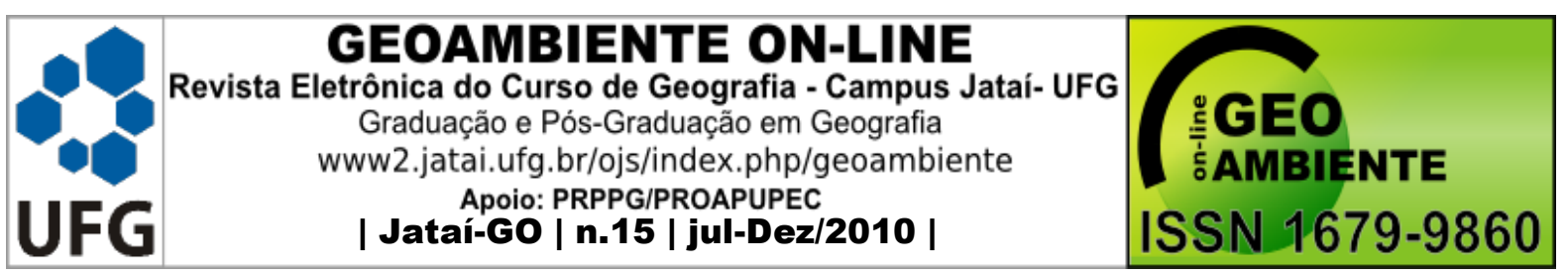

Abasto Sur. Asociación Latinoamericana de Producción Animal (ALPA), Uruguay, 2000, CD ROM.

BUSTAMANTE, C. La zonificación ambiental como estrategia para la planificación de sistemas agroforestales y la recuperación y conservación de los recursos naturales. En Simposio Internacional Sistemas Agroforestales Pecuarios de América del Sur. Brasil, ISBN 92-5-904475-8, 7pp, 2002.

BEAULIEU, N.; JARAMILLO, J.; LECLEC, G.; PABÓN, S. y GÓMEZ QUIRÓS, C.A. Propuesta metodológica para el componente participativo del ordenamiento territorial, basada en el desarrollo de una visión común del futuro. CIAT. Cali, Colombia, p.12., 2000.

BERTOT, J. A.; VÁZQUEZ, R.; DE LA TORRE, R. y COLLANTES, M. Estimación de los nacimientos y las pérdidas económicas por baja eficiencia reproductiva en rebaños lecheros en las condiciones de Camagüey. Rev. Prod. anim. 18 (2), 2006.

CHAMICHUMBI, W. Ordenamiento de las cuencas hidrográficas de la región andina. Consultado el 13 de febrero de 2007 en: (http://www.ecoportal.net).

DE LA COLINA, A. J. Desafíos y perspectivas de la ganadería vacuna en el desarrollo rural sostenible en América Latina y Cuba. En IV Taller Científico Internacional "El medio rural en el nuevo milenio: retos y perspectivas", La Habana, Cuba, 2005, CD ROM.

DIRECCIÓN NACIONAL DE CUENCAS HIDROGRÁFICAS, Metodología simplificada para el diagnóstico y la gestión en las cuencas hidrográficas. Instituto Nacional de Recursos Hidráulicos, La Habana, Cuba, 17pp, 2006.

DOUROJEANNI, A., Creación de entidades de cuencas en América Latina y el Caribe. Santa Cruz del Rosario. CEPAL, 33pp, 1997.

FAO. Conferencia sobre agua para alimentos y ecosistemas: Para que sea una realidade!. Consultado el 4 fr mrzo de 2007 en (http://www.fao.org/ag/wfe2005/dics/LEADwater.es).

FRÓMETA, A.; C. RIVIAUX y VILLAMET, P. Aplicación de técnicas para el manejo integral de cuencas hidrográficas con énfasis en suelos y agua de la cuenca Guantánamo Guaso local Hondones. En III Congreso Forestal de Cuba. La Habana, Cuba, ISBN 959-246119-8, 2004. 


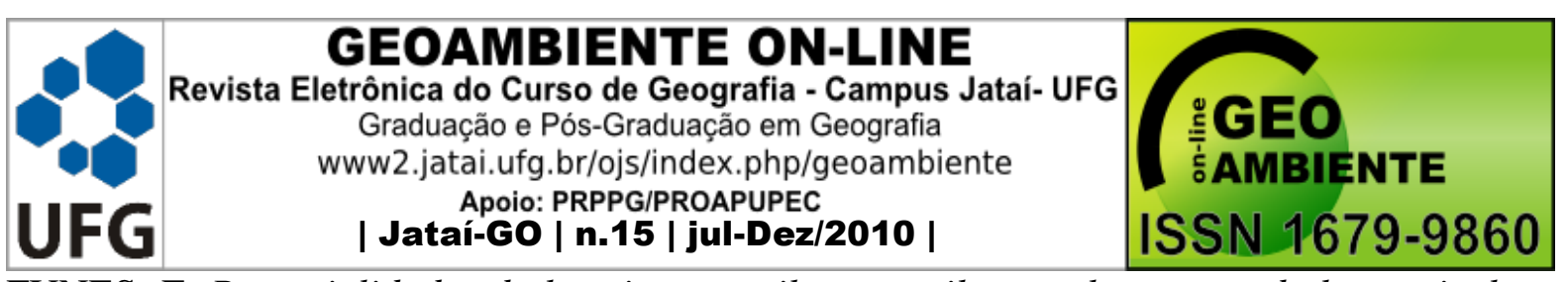

FUNES, F. Potencialidades de los sistemas silvopastoriles en el contexto de la agricultura orgánica. En Memorias del V Taller Internacional Silvopastoril y I Reunión Regional de Morera, ISBN 959-16-0172-7. Matanzas, Cuba. 2002.

GACETA OFICIAL DE LA REPÚBLICA DE CUBA, MINISTERIO DE JUSTICIA. Edición Extraordinaria, La Habana, 11 de Julio de 1997, Año XCV. Ley 81 del Medio Ambiente. Número 7, 47p., 1997.

GÓMEZ-OREA, D. Evaluación del Impacto Ambiental. Editorial Agrícola Española S. A. España, 702 pp., 1999.

GUEVARA, G.V. Valoración de sistemas lecheros cooperativos de la cuenca Camagüey Jimaguayú. Tesis presentada en opción al grado de Doctor en Ciencias Veterinarias. Universidad de Camagüey, Facultad de Ciencias Agropecuarias, 80pp., 2005.

HARVEY, C.A., F. ALPIZAR, M. CHACÓN y MADRIGAL, R. Assessing linkages between Agriculture and Biodiversity in Central America: Historical overview and Future perspectives. Mesoamerican and Caribbean Region, Conservation Science Program. The Nature Conservancy (TNC), San José, Costa Rica, 140 pp., 2005.

IBRAHIM, M. y MORA, J. Criterios y herramientas para la promoción de una ganadería eco - amigable en el trópico americano. En Memorias del curso-taller internacional “Ganadería, Desarrollo Sostenible y medio Ambiente”. La Habana, Cuba, p 23 - 28, 2003.

IBRAHIM, M.; C. VILLANUEVA; F. CASASOLA y ROJAS, J. Sistemas silvopastoriles como una herramienta para el mejoramiento de la productividad y restauración de la integridad ecológica de paisajes ganaderos. En IV Congreso de Agroforestería Pecuaria Sostenible. Matanzas, Cuba, ISBN 959 - 16 - 0478 - 5, 2006.

JEREZ, I. Comportamiento de vacas lecheras con diferentes cargas en gramíneas tropicales. Tesis en opción al grado de Dr. En Ciencias Veterinarias, Universidad Agraria de La Habana, ICA, Cuba, 87pp., 1983.

CUBA - MINISTERIO DE CIENCIA, TECNOLOGÍA Y MEDIO AMBIENTE Estrategia Nacional Ambiental de Cuba, 67pp., 1997.

, Situación Ambiental de la Provincia de Camagüey. Informe de la Unidad de Medio Ambiente, Delegación Territorial del CITMA en Camagüey, 125pp., Cuba, 2004. 


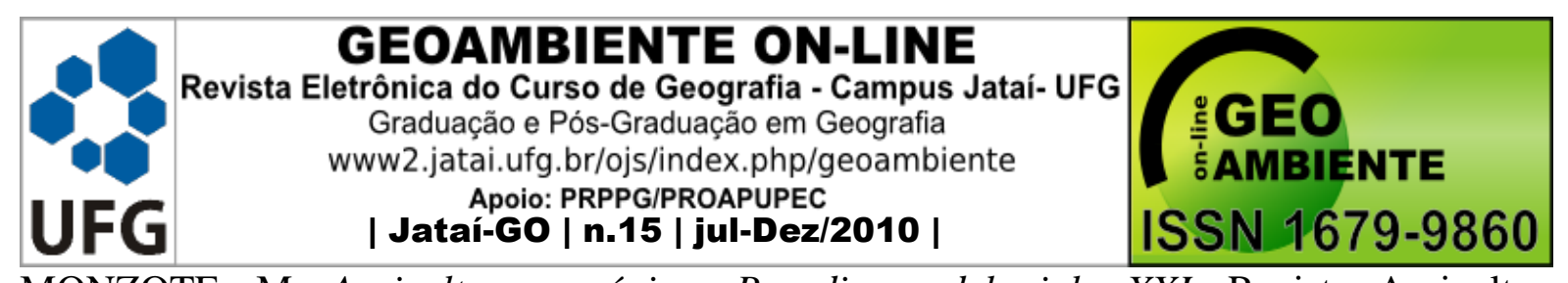

MONZOTE, M. Agricultura orgánica: Paradigma del siglo XXI. Revista Agricultura Orgánica. Año 6(1): 7-10, 2000.

MURGUEITIO, E. Impacto ambiental de la ganadería de leche en Colombia y alternativas de solución. Livestock Research for Rural Development 15(10): 1-16, 2003.

ODUM, E.P. Ecología. Tercera edición. Ed. Revolucionarias, 639 pp., 1987.

PASTAKIA, C.M.R. The Rapid Impact Assessment Matrix (RIAM) - A New Tool for Environmental Impact Assessment, In Kurt Jensen (ed.), Environmental Impact Assessment using the Rapid Impact Assessment Matrix (RIAM), Olsen \& Olsen, Fredensborg, Denmark, p. 8-18, 2002.

PRIMELLES, J; ZEQUEIRA, M.E.; ACOSTA, Z; MARTÍN, G.; FERRER, A.; BRITO, O; HERNÁNDEZ, L.; LI, M.E.; FRANCIS, D.; LIAZ, A.; MACHADO, W.; AGUILAR, J.; PLASENCIA, J.M.; GODÍNEZ, D.; ENRÍQUEZ, N. y CEBRIAN, A. Monografía del Diagnóstico Ambiental de la Cuenca del río San Pedro. Centro de Investigaciones de Medio Ambiente de Camagüey. Ministerio de Ciencia, Tecnología y Medio Ambiente 161pp., 2003.

RAMÍREZ, B.L. Caracterización y alternativas productivas para fincas ganaderas establecidas en la Amazonía Colombiana. Tesis en opción al Grado de Dr. en Ciencias Agropecuarias. Centro Agronómico Tropical de Investigación y Enseñanza. Costa Rica, 127 pp., 2002.

REYES LÓPEZ, A. Propuesta de manejo pecuario para desarrollo integral de microcuencas. En Memorias IX Congreso Regional de Irrigación. Simposio de Manejo Integral de Cuencas Hidrográficas. Culiacán, México, p.156-161, 1999.

REYES, J. Efecto de las altas cargas y el manejo de la intensidad de pastoreo, en el sistema suelo-planta-animal en condiciones de bajos insumos. Tesis en opción al grado científico de Doctor en Ciencias Veterinarias. ICA-Universidad Agraria del La Habana, 104pp., 2003.

RODRÍGUEZ CASTELLÓN, S. Consideraciones sobre el desarrollo agrario y el medio ambiente en las condiciones de Cuba. En IV Taller Científico Internacional "El medio rural en el nuevo milenio: retos y perspectivas”. La Habana, Cuba, 25pp., 2005.

SENRA, A. Características y aspectos fundamentales del Pastoreo Racional Voisín y consideraciones relacionadas con su uso. Conferencia, Instituto de Ciencia Animal. La Habana, Cuba, 30pp., 1993. 


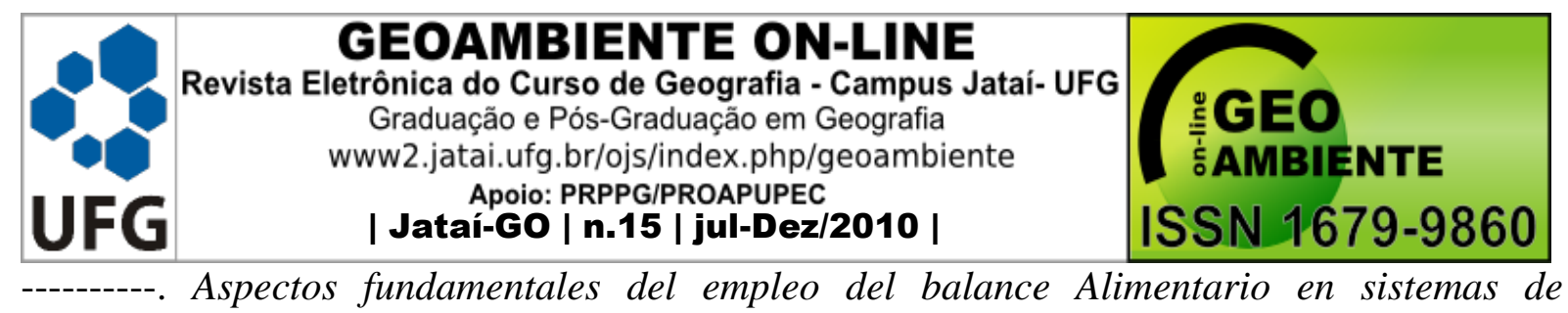

producción de leche a base de pastos. En Manual temas de Tecnologías Ganaderas. ACPA. INTERMON. Ciudad de La Habana, Cuba, 44pp., 2001.

SYSTAT 10.2. SYSTAT Software Insurance. U.S.A., 2002.

VILLASUSO, I.M. Las cuencas hidrográficas como unidades básicas de gestión ambiental y de referencia para el manejo integrado en pos del desarrollo sostenible. Estudio de caso río Yumurí Matanzas, Cuba. En III Congreso Forestal de Cuba. La Habana, Cuba, ISBN 959246-119-8, 2004. 